\title{
Some T-Spherical Fuzzy Einstein Interactive Aggregation Operators and Their Application to Selection of Photovoltaic Cells
}

\author{
Shouzhen Zeng (iD), ${ }^{1,2,3}$ Muhammad Munir, ${ }^{4}$ Tahir Mahmood $\left(\mathbb{D},{ }^{4}\right.$ and Muhammad Naeem ${ }^{4}$ \\ ${ }^{1}$ School of Business, Ningbo University, Ningbo 315211, China \\ ${ }^{2}$ College of Statistics and Mathematics, Zhejiang Gongshang University, Hangzhou 310018, China \\ ${ }^{3}$ School of Management, Fudan University, Shanghai 200433, China \\ ${ }^{4}$ Department of Mathematics and Statistics, International Islamic University, Islamabad, Pakistan \\ Correspondence should be addressed to Tahir Mahmood; tahirbakhat@iiu.edu.pk
}

Received 12 April 2020; Accepted 11 May 2020; Published 9 June 2020

Guest Editor: Zaoli Yang

Copyright (c) 2020 Shouzhen Zeng et al. This is an open access article distributed under the Creative Commons Attribution License, which permits unrestricted use, distribution, and reproduction in any medium, provided the original work is properly cited.

\begin{abstract}
In this article, it is pointed out that the existing intuitionistic fuzzy and T-spherical fuzzy Einstein averaging and geometric operators have some limitations. To overcome these limitations, we proposed some new averaging and geometric operators in the T-spherical fuzzy environment instead of the intuitionistic fuzzy environment because the T-spherical fuzzy set is the most generalized form and the proposed operators can be particularized to the intuitionistic fuzzy environment. First, new operational laws for T-spherical fuzzy information are defined, on the basis of which Einstein geometric interaction operators and Einstein averaging interactive aggregation operators are then proposed. Some basic properties and advantages of proposed aggregation operators are also discussed. Moreover, the proposed operators are applied to the MADM problem to check their reliability. The superiority of the proposed operators over existing work is checked with the help of an example.
\end{abstract}

\section{Introduction}

To deal with uncertainty, a tool called fuzzy set (FS) was introduced by Zadeh [1]. FS tells the degree of membership (MD) of an object. Intuitionistic fuzzy set (IFS), a generalization of FS, was introduced by Atanassov [2, 3]. IFS has two functions, a membership and a nonmembership function, and both functions map on a closed unit interval from a nonempty set. As the name indicates that they tell the MD and nonmembership degree (NMD) of an object, respectively, the IFS has a condition that the sum of both degrees must belong to $[0,1]$. Whenever the sum of both degrees exceeds from 1 , IFS fails to handle that information. To overcome this issue, Yager $[4,5]$ relaxed the condition that the square sum of both degrees must belong to $[0,1]$ and named that structure as Pythagorean fuzzy set (PyFS). In PyFS, the decision maker has more options for giving values to an object but the decision maker is not free to give any value because there are some limitations. To overcome this problem, Yager [6] proposed an improved fuzzy structure called q-rung orthopair fuzzy set (qROPFS) in which there is no limitation overall.
The notion picture fuzzy set (PFS) was introduced by Coung [7]. In PFS, there are four functions which express the $\mathrm{MD}$, NMD, abstinence (AD), and refusal with a condition that the sum of $\mathrm{MD}, \mathrm{AD}$, and NMD must belong to $[0,1]$. PFS fails when their sum exceeds one. Mahmood et al. [8] overcame this problem by introducing the concept of spherical fuzzy set in which they relaxed the condition that the square sum of $\mathrm{MD}, \mathrm{AD}$, and $\mathrm{NMD}$ must belong to $[0,1]$. In [8], they also proposed the concept of a T-spherical fuzzy set in which the decision makers have to find the power for which the sum lies in the unit's closed interval.

All the fuzzy structures discussed above are used to solve many problems in the field of decision making, similarity measures, etc. Xu and Yager [9] proposed weighted geometric operators for IFS. Zeng et al. [10] proposed some probabilistic averaging operators for IFS and studied their application to group decision making. Garg [11] investigated the MADM problem using interval-valued IF robust geometric aggregation operators. Zhang et al. [12] discussed the IF MULTIMOORA approach for the MADM problem. He et al. [13] investigated the MADM problem using interactive geometric 
aggregation operators for IFS. Zhao and Wei [14] used Einstein $\mathrm{t}$-norm and $\mathrm{t}$-conorm to introduce IF Einstein geometric aggregation (IFEWA) operators and IF Einstein averaging aggregation (IFEWG) operators to solve the MADM problem. $\mathrm{Mu}$ et al. [15] investigated the MADM problem using Zhenyuan aggregation operators for intervalvalued IFSs. Some MADM problems are solved for IFSs in [16-18]. Garg [19] investigated the MADM problem using Einstein norms for PyFSs. Wei and $\mathrm{Lu}$ [20] used power operators for PyFSs to solve the MADM problem. Yang and Chang [21] solved the MADM problem using interval-valued Pythagorean normal fuzzy aggregation operators. Zeng et al. [22] investigated the MADM problem using the novel aggregation method for PyFS. Zeng et al. [23] investigated the MADM problem using PyF confidence aggregation operators. By using PyFSs, some problems are solved in [24, 25]. Peng et al. [26] investigated the MADM problem by using exponential aggregation operators for q-ROPFS. Yang et al. [27] solved the MADM problem using q-ROPF interaction Heronian mean operators. Yang et al. [28] proposed the q-ROPF multicriteria decision algorithm and used it to investigate multiple heterogeneous relationships. Hussain et al. [29] investigated the MADM problem using a covering-based q-ROPF rough set model. Hussain et al. [30] used q-ROPF soft averaging operators to solve the MADM problem. Some MADM problems are studied using q-ROPFSs in [31-33].

Wei [34] proposed picture fuzzy Hamacher aggregation operators and studied their application in the MADM problem. Khan et al. [35] investigated the MADM problem using some logarithmic aggregation operators for PFS. Some MADM problems for PFS are studied in [36-38]. Quek et al. [39] studied the MADM problem using T-spherical fuzzy weighted aggregation operators. Garg [40] proposed interactive aggregation operators for T-spherical fuzzy sets and solved the MADM problem using the proposed operators. Zeng et al. [41] proposed immediate probabilistic averaging aggregation operators for T-spherical fuzzy sets and investigated their application in the selection of solar cells. Munir et al. [42] solved the MADM problem using Einstein aggregation operators for T-spherical fuzzy set. By using spherical fuzzy sets and T-spherical fuzzy sets, some MADM problems are studied in [43-46].

The existing Einstein operations $[14,42]$ fail under some circumstances, e.g., if $I_{1}=\left(m_{A}, 0\right)$ and $I_{2}=\left(0, n_{A}\right)$ are intuitionistic fuzzy numbers (IFNs), then IFEWA operators aggregate these IFNs as (some value, 0 ) and IFEWG operators aggregates these IFNs as (0, some value). From the above example, it is easy to notice that the IFEWA operator cannot aggregate the nonmembership value when nonmembership value of anyone IFN becomes zero and similarly the IFEWG operator cannot aggregate the membership value when nonmembership value of anyone IFN becomes zero. This issue motivates us to propose some improved Einstein aggregation operators which will overcome these problems.

The purposes of this manuscript are as follows:

(1) To develop some new interactive Einstein operational laws
(2) To develop new T-spherical fuzzy Einstein weighted geometric interaction operators using the new proposed interactive operational laws

(3) To develop new T-spherical fuzzy Einstein weighted averaging interaction operators based on the new proposed interactive operations

(4) To present an algorithm for solving the MADM problem

(5) To check the applicability of the proposed operators through a numerical example

To achieve these aims, the rest of the article is summarized as follows. Section 2 has some notions that will be helpful for the readers in further sections. In Section 3, new operational laws are proposed for T-spherical fuzzy sets, and in Section 4, T-spherical fuzzy Einstein interaction geometric operators are proposed and some basic properties are also proved. In Section 5, T-spherical fuzzy Einstein interaction averaging operators are defined and some of their basic properties are also discussed. The advantages of the proposed work and comparative analysis between the existing and proposed operators are developed in Section 6. In Section 7, an algorithm for solving a MADM problem is developed and also applied in an example. A brief conclusion of the manuscript is included in Section 8.

\section{Preliminaries}

Some basic definitions over the set $X$ are defined in this section. These basic notions will help the readers to understand the proposed work.

Definition 1 (see [8]). A T-spherical fuzzy set on $X$ is defined as

$$
P=\{(x, m(x), i(x), n(x)) \mid x \in X\}
$$

where $m, i, n: X \longrightarrow[0,1]$ are a membership, abstinence, and nonmembership function, respectively, with the condition $0 \leq m^{t}(x)+i^{t}(x)+n^{t}(x) \leq 1, \forall x \in X, t \in \mathbb{N}$.

$r(x)=\sqrt[t]{1-\left(m^{t}(x)+i^{t}(x)+n^{t}(x)\right)}$ is called the refusal degree of $x$ in $P$, and $(m, i, n)$ is known as T-spherical fuzzy number (T-SFN).

Definition 2 (see [8]). Let $P=(m, i, n) \in$ T-spherical fuzzy set. Then, the score value of $P$ is defined as SC $(P)=m^{t}(x)-n^{t}(x)$, and the accuracy value of $P$ is defined as $\mathrm{AC}(P)=m^{t}(x)+i^{t}(x)+n^{t}(x)$. A T-SFN with a greater score is superior to others. If the score of any two T-SFNs becomes equal, then their superiority will be checked with the help of their accuracy values. The number which has greater accuracy value will be superior as compared to the others. If accuracy values also become equal, then both numbers are considered similar.

Remark 1

(1) Definitions 1 and 2 can be reduced for spherical fuzzy set if we consider $t=2$ 
(2) Definitions 1 and 2 can be reduced for PFS if we consider $t=1$

(3) Definitions 1 and 2 can be reduced for q-ROFS if we consider $i=0$

(4) Definitions 1 and 2 can be reduced for PyFS if we consider $t=2$ and $i=0$
(5) Definitions 1 and 2 can be reduced for IFS if we consider $t=1$ and $i=0$

(6) Definition 1 can be reduced for FS if we consider $t=1, i=0$, and $n=0$

Definition 3 (see [14]). Some operations for any two IFNs $I_{1}=\left(m_{1}, n_{1}\right)$ and $I_{2}=\left(m_{2}, n_{2}\right)$ are defined as

$$
\begin{aligned}
I_{1} \otimes I_{2} & =\left(\frac{m_{1} m_{2}}{1+\left(1-m_{1}\right)\left(1-m_{2}\right)}, \frac{n_{1}+n_{2}}{1+n_{1} n_{2}}\right), \\
I_{1} \oplus I_{2} & =\left(\frac{m_{1}+m_{2}}{1+m_{1} m_{2}}, \frac{n_{1} n_{2}}{1+\left(1+n_{1}\right)\left(1-n_{2}\right)}\right), \\
\tau I_{1} & =\left(\frac{\left(1+m_{1}\right)^{\tau}+\left(1-m_{1}\right)^{\tau}}{\left(1+m_{1}\right)^{\tau}-\left(1-m_{1}\right)^{\tau}}, \frac{2 n_{1}^{\tau}}{\left(2-n_{1}\right)^{\tau}+n_{1}^{\tau}}\right), \quad \tau>0, \\
I_{1}^{\tau} & =\left(\frac{2 m_{1}^{\tau}}{\left(2-m_{1}\right)^{\tau}+m_{1}^{\tau}}, \frac{\left(1+n_{1}\right)^{\tau}+\left(1-n_{1}\right)^{\tau}}{\left(1+n_{1}\right)^{\tau}-\left(1-n_{1}\right)^{\tau}}\right), \quad \tau>0 .
\end{aligned}
$$

\section{Einstein Interaction Operations for T-Spherical Fuzzy Set}

Existing Einstein operations have some limitations that they fail under some conditions. So, we proposed some new
Einstein operations on which we define some new aggregation operators. Let $P_{1}=\left(m_{1}, i_{1}, n_{1}\right)$ and $P_{2}=\left(m_{2}, i_{2}, n_{2}\right)$ be two T-spherical fuzzy sets; then, their Einstein operations are as follows:

$$
\begin{aligned}
& P_{1} \otimes P_{2}=\left(\begin{array}{c}
\sqrt[t]{\frac{2\left(\left(1-n_{1}^{t}-i_{1}^{t}\right)\left(1-n_{2}^{t}-i_{2}^{t}\right)-\left(1-m_{1}^{t}-i_{1}^{t}-n_{1}^{t}\right)\left(1-m_{2}^{t}-i_{2}^{t}-n_{2}^{t}\right)\right)}{\left(1+n_{1}^{t}\right)\left(1+n_{2}^{t}\right)+\left(1-n_{1}^{t}\right)\left(1-n_{2}^{t}\right)}}, \\
\sqrt[t]{\frac{\left(1+i_{1}^{t}\right)\left(1+i_{2}^{t}\right)-\left(1-i_{1}^{t}\right)\left(1-i_{2}^{t}\right)}{\left(1+i_{1}^{t}\right)\left(1+i_{2}^{t}\right)+\left(1-i_{1}^{t}\right)\left(1-i_{2}^{t}\right)}}, \sqrt[t]{\frac{\left(1+n_{1}^{t}\right)\left(1+n_{2}^{t}\right)-\left(1-n_{1}^{t}\right)\left(1-n_{2}^{t}\right)}{\left(1+n_{1}^{t}\right)\left(1+n_{2}^{t}\right)+\left(1-n_{1}^{t}\right)\left(1-n_{2}^{t}\right)}}
\end{array}\right), \\
& P_{1} \oplus P_{2}=\left(\begin{array}{c}
\sqrt[t]{\frac{\left(1+m_{1}^{t}\right)\left(1+m_{2}^{t}\right)-\left(1-m_{1}^{t}\right)\left(1-m_{2}^{t}\right)}{\left(1+m_{1}^{t}\right)\left(1+m_{2}^{t}\right)+\left(1-m_{1}^{t}\right)\left(1-m_{2}^{t}\right)}}, \sqrt[t]{\frac{\left(1+i_{1}^{t}\right)\left(1+i_{2}^{t}\right)-\left(1-i_{1}^{t}\right)\left(1-i_{2}^{t}\right)}{\left(1+i_{1}^{t}\right)\left(1+i_{2}^{t}\right)+\left(1-i_{1}^{t}\right)\left(1-i_{2}^{t}\right)}}, \\
\sqrt[t]{\frac{2\left(\left(1-m_{1}^{t}-i_{1}^{t}\right)\left(1-m_{2}^{t}-i_{2}^{t}\right)-\left(1-m_{1}^{t}-i_{1}^{t}-n_{1}^{t}\right)\left(1-m_{2}^{t}-i_{2}^{t}-n_{2}^{t}\right)\right)}{\left(1+m_{1}^{t}\right)\left(1+m_{2}^{t}\right)+\left(1-m_{1}^{t}\right)\left(1-m_{2}^{t}\right)}}
\end{array}\right), \\
& \tau P_{1}=\left(\sqrt[t]{\frac{\left(1+m_{1}^{t}\right)^{\tau}-\left(1-m_{1}^{t}\right)^{\tau}}{\left(1+m_{1}^{t}\right)^{\tau}+\left(1-m_{1}^{t}\right)^{\tau}}}, \sqrt[t]{\frac{\left(1+i_{1}^{t}\right)^{\tau}-\left(1-i_{1}^{t}\right)^{\tau}}{\left(1+m_{1}^{t}\right)^{\tau}+\left(1-m_{1}^{t}\right)^{\tau}}}, \sqrt[t]{\frac{2\left(\left(1-m_{1}^{t}-i_{1}^{t}\right)^{\tau}-\left(1-m_{1}^{t}-i_{1}^{t}-n_{1}^{t}\right)^{\tau}\right)}{\left(1+m_{1}^{t}\right)^{\tau}+\left(1-m_{1}^{t}\right)^{\tau}}}\right), \quad \tau>0, \\
& \left(P_{1}\right)^{\tau}=\left(\sqrt[t]{\frac{2\left(\left(1-n_{1}^{t}-i_{1}^{t}\right)^{\tau}-\left(1-m_{1}^{t}-i_{1}^{t}-n_{1}^{t}\right)^{\tau}\right)}{\left(1+n_{1}^{t}\right)^{\tau}+\left(1-n_{1}^{t}\right)^{\tau}}}, \sqrt[t]{\frac{\left(1+i_{1}^{t}\right)^{\tau}-\left(1-i_{1}^{t}\right)^{\tau}}{\left(1+i_{1}^{t}\right)^{\tau}+\left(1-i_{1}^{t}\right)^{\tau}}}, \sqrt[t]{\frac{\left(1+n_{1}^{t}\right)^{\tau}-\left(1-n_{1}^{t}\right)^{\tau}}{\left(1+n_{1}^{t}\right)^{\tau}+\left(1-n_{1}^{t}\right)^{\tau}}}\right), \quad \tau>0 .
\end{aligned}
$$

\section{Remark 2}

(1) The defined operations will be reduced to spherical fuzzy sets for $t=2$

(2) The defined operations will be reduced to PFSs for $t=1$
(3) The defined operations will be reduced to q-ROFSs for $i=0$

(4) The defined operations will be reduced to PyFSs for $t=2$ and $i=0$

(5) The defined operations will be reduced to IFSs for $t=1$ and $i=0$ 
(6) The defined operations will be reduced to FSs for $t=1, i=0$, and $n=0$

\section{T-Spherical Fuzzy Einstein Geometric Interaction Operators}

In this section, on the basis of new proposed Einstein operations, we defined geometric interaction operators in the environment of T-spherical fuzzy set and some of its basic properties such as monotonicity, boundedness, and idempotency are also discussed. The validity of the proposed work is checked with the help of an example.

Definition 4. For any collection of T-SFNs, $P_{j}=\left(m_{j}, i_{j}, n_{j}\right)$ $(j=1,2,3, \ldots, k)$. The mapping

$\mathrm{T}-\operatorname{SFEWIG}_{w}\left(P_{1}, P_{2}, \ldots, P_{k}\right)=\otimes_{j=1}^{k} P_{j}^{w_{j}}$

$$
=\left(\begin{array}{c}
\sqrt[t]{\frac{2\left(\prod_{j=1}^{k}\left(1-n_{j}^{t}-i_{j}^{t}\right)^{w_{j}}-\prod_{j=1}^{k}\left(1-m_{j}^{t}-i_{j}^{t}-n_{j}^{t}\right)^{w_{j}}\right)}{\prod_{j=1}^{k}\left(1+n_{j}^{t}\right)^{w_{j}}+\prod_{j=1}^{k}\left(1-n_{j}^{t}\right)^{w_{j}}}}, \sqrt[t]{\frac{\prod_{j=1}^{k}\left(1+i_{j}^{t}\right)^{w_{j}}-\prod_{j=1}^{k}\left(1-i_{j}^{t}\right)^{w_{j}}}{\prod_{j=1}^{k}\left(1+i_{j}^{t}\right)^{w_{j}}+\prod_{j=1}^{k}\left(1-i_{j}^{t}\right)^{w_{j}}}}, \\
\sqrt[\prod_{j=1}^{k}\left(1+n_{j}^{t}\right)^{w_{j}}-\prod_{j=1}^{k}\left(1-n_{j}^{t}\right)^{w_{j}}]{\prod_{j=1}^{k}\left(1+n_{j}^{t}\right)^{w_{j}}+\prod_{j=1}^{k}\left(1-n_{j}^{t}\right)^{w_{j}}}
\end{array}\right),
$$

is called the T-spherical fuzzy Einstein weighted interactive geometric (T-SFEWIG) operator, where $w=\left(w_{1}, \ldots, w_{k}\right)^{T}$ is the weight vector of $P_{j}$ with $w_{j} \in[0,1]$ and $\sum_{j=1}^{k} w_{j}=1$.
Theorem 1. If all $P_{j}=P_{0}$, then T-SFEWIG $\left(P_{1}, P_{2}, \ldots, P_{k}\right)=P_{0}$.

Proof. Let $P_{j}=P_{0}=\left(m_{0}, i_{0}, n_{0}\right)$ for all $j$; then,

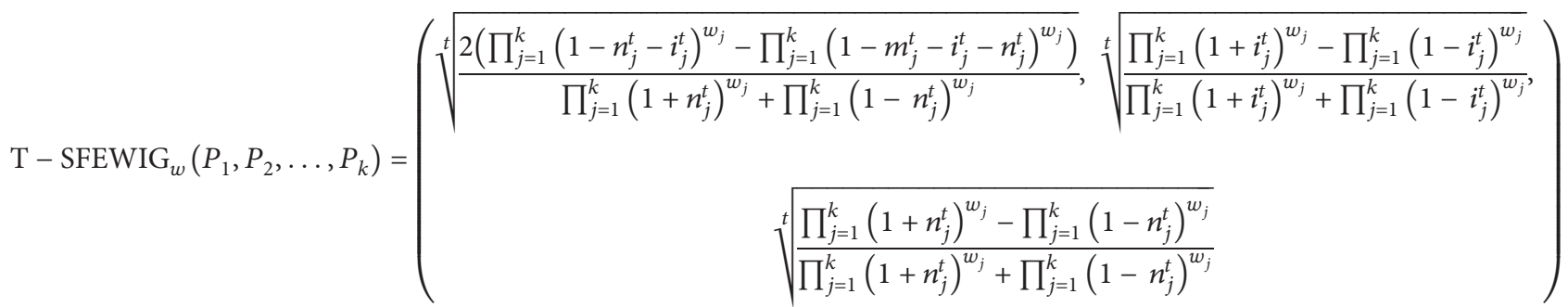

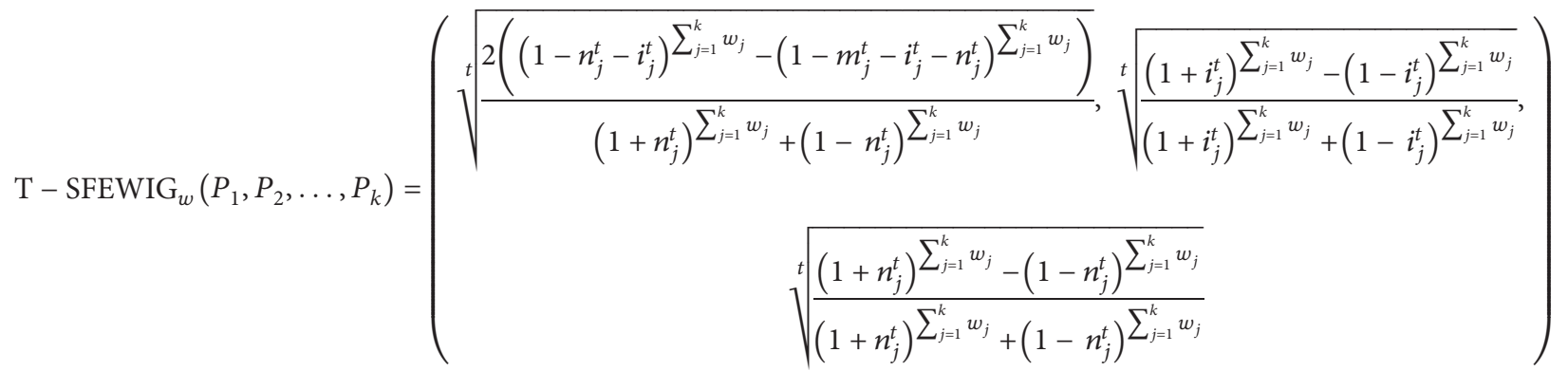

$$
\begin{aligned}
& =\left(m_{0}, i_{0}, n_{0}\right)=P_{0} .
\end{aligned}
$$

Theorem 2. Consider a collection of T-SFNs $P_{j}(j=1,2, \quad$ Proof. Straightforward. $\ldots, k)$ with $P^{L}=\min _{j} P_{j}$ and $P^{U}=\max _{j} P_{j}$. Then,

$$
P^{L} \leq \mathrm{T}-\operatorname{SFEWIG}_{w}\left(P_{1}, P_{2}, \ldots, P_{k}\right) \leq P^{U} .
$$

Theorem 3. Consider any two T-SFNs $P_{j}=\left(m_{j}, i_{j}, n_{j}\right)$ and $P_{j}^{\prime}=\left(m_{j}^{\prime}, i_{j}^{\prime}, n_{j}^{\prime}\right)(j=1,2, \ldots, k)$ such that $P_{j} \leq P_{j}^{\prime}$ for all $j$. Then, 
$\mathrm{T}-\operatorname{SFEWIG}_{w}\left(P_{1}, P_{2}, \ldots, P_{k}\right) \leq \mathrm{T}-\operatorname{SFEWIG}_{w}\left(P_{1}^{\prime}, P_{2}^{\prime}, \ldots, P_{k}^{\prime}\right)$.
Proof. Let $P_{j} \leq P_{j}^{\prime}$; then, $m_{j} \leq m_{j}^{\prime}, i_{j} \leq i_{j}^{\prime}$, and $n_{j} \geq n_{j}^{\prime}$. Then, by using basic information,

$$
\begin{aligned}
& \sqrt[t]{\frac{2\left(\prod_{j=1}^{k}\left(1-n_{j}^{t}-i_{j}^{t}\right)^{w_{j}}-\prod_{j=1}^{k}\left(1-m_{j}^{t}-i_{j}^{t}-n_{j}^{t}\right)^{w_{j}}\right)}{\prod_{j=1}^{k}\left(1+n_{j}^{t}\right)^{w_{j}}+\prod_{j=1}^{k}\left(1-n_{j}^{t}\right)^{w_{j}}}} \\
& \leq \sqrt[t]{\frac{2\left(\prod_{j=1}^{k}\left(1-\left(n_{j}^{\prime}\right)^{t}-\left(i_{j}^{\prime}\right)^{t}\right)^{w_{j}}-\prod_{j=1}^{k}\left(1-\left(m_{j}^{\prime}\right)^{t}-\left(i_{j}^{\prime}\right)^{t}-\left(n_{j}^{\prime}\right)^{t}\right)^{w_{j}}\right)}{\prod_{j=1}^{k}\left(1+\left(n_{j}^{\prime}\right)^{t}\right)^{w_{j}}+\prod_{j=1}^{k}\left(1-\left(n_{j}^{\prime}\right)^{t}\right)^{w_{j}}}} \\
& \sqrt[t]{\sqrt[t]{\frac{\prod_{j=1}^{k}\left(1+i_{j}^{t}\right)^{w_{j}}-\prod_{j=1}^{k}\left(1-i_{j}^{t}\right)^{w_{j}}}{\prod_{j=1}^{k}\left(1+i_{j}^{t}\right)^{w_{j}}+\prod_{j=1}^{k}\left(1-i_{j}^{t}\right)^{w_{j}}}} \leq \sqrt{\frac{\left.\prod_{j=1}^{k}\left(1+\left(i_{j}^{\prime}\right)^{t}\right)^{w_{j}}-\prod_{j}^{t}\right)^{w_{j}}+\prod_{j=1}^{k}\left(1-n_{j=1}^{k}\left(1-n_{j}^{t}\right)^{w_{j}}\right.}{\left.\prod_{j=1}^{k}\left(1+\left(i_{j}^{\prime}\right)^{t}\right)^{w_{j}}+\prod_{j=1}^{k}\left(1-\left(i_{j}^{\prime}\right)^{t}\right)^{t}\right)^{w_{j}}}} \geq \sqrt[t]{\frac{\prod_{j=1}^{k}\left(1+\left(n_{j}^{\prime}\right)^{t}\right)^{w_{j}}-\prod_{j=1}^{k}\left(1-\left(n_{j}^{\prime}\right)^{t}\right)^{w_{j}}}{\prod_{j=1}^{k}\left(1+\left(n_{j}^{\prime}\right)^{t}\right)^{w_{j}}+\prod_{j=1}^{k}\left(1-\left(n_{j}^{\prime}\right)^{t}\right)^{w_{j}}}}} .
\end{aligned}
$$

This shows that

$\mathrm{T}-\operatorname{SFEWIG}_{w}\left(P_{1}, P_{2}, \ldots, P_{k}\right) \leq \mathrm{T}-\operatorname{SFEWIG}_{w}\left(P_{1}, P_{2}, \ldots, P_{k}\right)$.
Definition 5. For any collection of T-SFNs, $P_{j}=\left(m_{j}, i_{j}, n_{j}\right)$ $(j=1,2,3, \ldots, k)$. The mapping

$\mathrm{T}-\operatorname{SFEOWIG}_{\omega}\left(P_{1}, P_{2}, \ldots, P_{k}\right)=\otimes_{j=1}^{k} P_{\sigma(j)}^{\omega_{j}}$

$$
=\left(\begin{array}{c}
\sqrt[t]{\frac{2\left(\prod_{j=1}^{k}\left(1-n_{\sigma(j)}^{t}-i_{\sigma(j)}^{t}\right)^{w_{j}}-\prod_{j=1}^{k}\left(1-m_{\sigma(j)}^{t}-i_{\sigma(j)}^{t}-n_{\sigma(j)}^{t}\right)^{w_{j}}\right)}{\prod_{j=1}^{k}\left(1+n_{\sigma(j)}^{t}\right)^{w_{j}}+\prod_{j=1}^{k}\left(1-n_{\sigma(j)}^{t}\right)^{w_{j}}}, \sqrt[t]{\frac{\prod_{j=1}^{k}\left(1+i_{\sigma(j)}^{t}\right)^{w_{j}}-\prod_{j=1}^{k}\left(1-i_{\sigma(j)}^{t}\right)^{w_{j}}}{\prod_{j=1}^{k}\left(1+i_{\sigma(j)}^{t}\right)^{w_{j}}+\prod_{j=1}^{k}\left(1-i_{\sigma(j)}^{t}\right)^{w_{j}}}}} \\
\sqrt[t]{\frac{\prod_{j=1}^{k}\left(1+n_{\sigma(j)}^{t}\right)^{w_{j}}-\prod_{j=1}^{k}\left(1-n_{\sigma(j)}^{t}\right)^{w_{j}}}{\prod_{j=1}^{k}\left(1+n_{\sigma(j)}^{t}\right)^{w_{j}}+\prod_{j=1}^{k}\left(1-n_{\sigma(j)}^{t}\right)^{w_{j}}}}
\end{array},\right.
$$

$\mathrm{T}-\mathrm{SFEOWIG}_{\omega}$ is called the T-spherical fuzzy Einstein ordered weighted interactive geometric (T-SFEOWIG) operator, where $\omega=\left(\omega_{1}, \ldots, \omega_{k}\right)^{T}$ is the associated weight vector of $P_{j}$ with $\omega_{j} \in[0,1]$ and $\sum_{j=1}^{k} \omega_{j}=1$ and $\sigma(j)$ is any permutation of $(1,2, \ldots, k)$ such that $\widetilde{P}_{\sigma(j-1)} \geq \widetilde{P}_{\sigma(j)}$.

Theorem 4. If all $P_{j}=P_{0}$, then T-SFEOWIG $\left(P_{1}, P_{2}, \ldots, P_{k}\right)=P_{0}$.

Proof. Same as in Theorem 1.
Theorem 5. Consider a collection of T-SFNs $P_{j}(j=$ $1,2, \ldots, k)$ with $P^{L}=\min _{j} P_{j}$ and $P^{U}=\max _{j} P_{j}$. Then,

$$
P^{L} \leq \mathrm{T}-\operatorname{SFEOWIG}_{\omega}\left(P_{1}, P_{2}, \ldots, P_{k}\right) \leq P^{U} .
$$

Proof. Straightforward.

Theorem 6. For any two T-SFNs, $P_{j}=\left(m_{j}, i_{j}, n_{j}\right)$ and $P_{j}^{\prime}=$ $\left(m_{j}^{\prime}, i_{j}^{\prime}, n_{j}^{\prime}\right)$ for all $(j=1,2, \ldots, k)$ such that $P_{j} \leq P_{j}^{\prime}$ for all $j$. Then, 
$\mathrm{T}-\operatorname{SFEOWIG}_{\omega}\left(P_{1}, P_{2}, \ldots, P_{k}\right) \leq \mathrm{T}-\mathrm{SFEOWIG}_{\omega}$

$$
\cdot\left(P_{1}^{\prime}, P_{2}^{\prime}, \ldots, P_{k}^{\prime}\right) \text {. }
$$

Proof. Directly follows from the proof of Theorem 3.

Definition 6. For any collection of T-SFNs, $P_{j}=\left(m_{j}, i_{j}, n_{j}\right)(j=1,2,3, \ldots, k)$. The mapping

$\mathrm{T}-\operatorname{SFEHIG}{ }_{w, \omega}\left(\widetilde{P}_{1}, \widetilde{P}_{2}, \ldots, \widetilde{P}_{k}\right)=\otimes_{j=1}^{k} \widetilde{P}_{\sigma(j)}^{\omega_{j}}$

$$
=\left(\begin{array}{c}
\sqrt{\frac{2\left(\prod_{j=1}^{k}\left(1-\widetilde{n}_{\sigma(j)}^{t}-\widetilde{i}_{\sigma(j)}^{t}\right)^{w_{j}}-\prod_{j=1}^{k}\left(1-\widetilde{m}_{\sigma(j)}^{t}-\widetilde{i}_{\sigma(j)}^{t}-\widetilde{n}_{\sigma(j)}^{t}\right)^{w_{j}}\right)}{\prod_{j=1}^{k}\left(1+n_{\sigma(j)}^{t}\right)^{w_{j}}+\prod_{j=1}^{k}\left(1-n_{\sigma(j)}^{t}\right)^{w_{j}}}, \sqrt[t]{\frac{\prod_{j=1}^{k}\left(1+\widetilde{i}_{\sigma(j)}^{t}\right)^{w_{j}}-\prod_{j=1}^{k}\left(1-\widetilde{i}_{\sigma(j)}^{t}\right)^{w_{j}}}{\prod_{j=1}^{k}\left(1+\widetilde{i}_{\sigma(j)}^{t}\right)^{w_{j}}+\prod_{j=1}^{k}\left(1-\widetilde{i}_{\sigma(j)}^{t}\right)^{w_{j}}},}} \\
\sqrt[t]{\frac{\prod_{j=1}^{k}\left(1+\widetilde{n}_{\sigma(j)}^{t}\right)^{w_{j}}-\prod_{j=1}^{k}\left(1-\widetilde{n}_{\sigma(j)}^{t}\right)^{w_{j}}}{\prod_{j=1}^{k}\left(1+\widetilde{n}_{\sigma(j)}^{t}\right)^{w_{j}}+\prod_{j=1}^{k}\left(1-\widetilde{n}_{\sigma(j)}^{t}\right)^{w_{j}}}}
\end{array},\right.
$$

$\mathrm{T}-$ SFEHIG $_{w, \omega}$ is called the T-spherical fuzzy Einstein hybrid interactive geometric (T-SFEHIG) operator, where $\widetilde{P}_{j}=\left(P_{j}\right)^{k w_{j}}$. Let $w=\left(w_{1}, \ldots, w_{k}\right)^{T}$ is the weight vector and $\omega=\left(\omega_{1}, \ldots, \omega_{k}\right)^{T}$ is the associated weight vector of $P_{j}$ with a condition that both weight and associated weight vectors belong to closed unit interval and their sum is equal to 1 .

Hybrid aggregation operators first aggregate the given data considering their attributes and then rearrange them in a specific order. After that, they aggregate the data considering their order. This means that hybrid operators are a generalization of weighted and ordered weighted operators. So, the T-SFEHIG operator will satisfy idempotency, monotonicity, and bounded property.

Example 1. Consider T-SFNs $P_{1}=(0.7,0.3,0.2), P_{2}=(0.9$, $0.1,0.6), P_{3}=(0.4,0.6,0.8), P_{4}=(0.1,0.5,0.7)$, and $P_{5}=$ $(0.0,0.0,0.8)$ with a weight vector $w=(0.25,0.20,0.15$, $0.18,0.22)^{T}$.

Solution 1. First of all, we find the aggregated value of these T-SFNs by using the T-spherical fuzzy Einstein hybrid geometric aggregation (T-SFEHG) operator [42] to find out the drawbacks of the given operators. For this purpose, first, we have to calculate the value of $t$ for which the given data lie in T-SF information.

$$
\begin{aligned}
& \text { As } 0.9+0.1+0.6=1.6 \\
& \text { For } t=2,0.9^{2}+0.1^{2}+0.6^{2}=1.18 \\
& t=3,0.9^{3}+0.1^{3}+0.6^{3}=0.946
\end{aligned}
$$

Similarly, for $t=3$, all the given data lie in the T-spherical fuzzy information.

By using T-spherical fuzzy Einstein weighted geometric operator, we shall be able to find these values:

$$
\begin{aligned}
& P_{1}=(0.6388,0.3232,0.5381), \\
& P_{2}=(0.9,0.1,0.6), \\
& P_{3}=(0.4163,0.5464,0.7370), \\
& P_{4}=(0.1050,0.4829,0.6776), \\
& P_{5}=(0.0,0.0,0.8206) .
\end{aligned}
$$

Their scores values will be

$$
\begin{aligned}
& \operatorname{SC}\left(P_{1}\right)=0.1048, \\
& \operatorname{SC}\left(P_{2}\right)=0.5130, \\
& \operatorname{SC}\left(P_{3}\right)=-0.3282, \\
& \operatorname{SC}\left(P_{4}\right)=-0.3099, \\
& \operatorname{SC}\left(P_{5}\right)=-0.5525 .
\end{aligned}
$$

Now using the score value, the aggregated values obtained by using T-SFEWG operators are rearranged in descending order. Then, these ordered values are again aggregated by using the T-SFEHG operator with associated weight vector being $\omega=(0.112,0.236,0.304,0.236,0.112)$ :

$$
\begin{aligned}
& \widetilde{P}_{\sigma(1)}=(0.9,0.1,0.6), \\
& \widetilde{P}_{\sigma(2)}=(0.6388,0.3232,0.5381), \\
& \widetilde{P}_{\sigma(3)}=(0.1050,0.4829,0.6776), \\
& \widetilde{P}_{\sigma(4)}=(0.4163,0.5464,0.7370), \\
& \widetilde{P}_{\sigma(5)}=(0.0,0.0,0.8206) .
\end{aligned}
$$

Now, employ the T-SFEHG operator as follows:

$$
\mathrm{T}-\operatorname{SFEHG}_{w, \omega}\left(P_{1}, \ldots, P_{5}\right)=(0.0,0.8525,0.9882) .
$$


From the above result, it is noticed that when abstinence or nonmembership value of one T-SFN is zero, then the T-SFEHG operator cannot aggregate the whole membership value. This shows a big flaw in the T-SFEHG operator. This means that the results obtained from T-SFEHG operators are not reliable. Now, by using the T-SFEHIG operator, we shall show that the proposed operator will overcome this drawback.

By using the T-SFEWIG operator, we shall be able to find

$$
\begin{aligned}
& P_{1}=(0.7393,0.3232,0.2154), \\
& P_{2}=(0.9,0.1,0.6), \\
& P_{3}=(0.4131,0.5464,0.7370), \\
& P_{4}=(0.0988,0.4829,0.6776), \\
& P_{5}=(0.0,0.0,0.8206) .
\end{aligned}
$$

Their scores values will be

$$
\begin{aligned}
& \operatorname{SC}\left(P_{1}\right)=0.3941 \\
& \operatorname{SC}\left(P_{2}\right)=0.5130 \\
& \operatorname{SC}\left(P_{3}\right)=-0.3299 \\
& \operatorname{SC}\left(P_{4}\right)=-0.3101 \\
& \operatorname{SC}\left(P_{5}\right)=-0.5525
\end{aligned}
$$

Now using the score value, the aggregated values obtained by using T-SEWIG operators are rearranged in descending order. Then, these ordered values are again aggregated by using the T-SFEHIG operator with the associated weight vector being $\omega=(0.112,0.236,0.304$, $0.236,0.112)$ :

$$
\begin{aligned}
& \widetilde{P}_{\sigma(1)}=(0.9,0.1,0.6), \\
& \widetilde{P}_{\sigma(2)}=(0.7393,0.3232,0.2154), \\
& \widetilde{P}_{\sigma(3)}=(0.0988,0.4829,0.6776), \\
& \widetilde{P}_{\sigma(4)}=(0.4131,0.5464,0.7370), \\
& \widetilde{P}_{\sigma(5)}=(0.0,0.0,0.8206) .
\end{aligned}
$$

Now, employ the T-SFEHG operator as follows:

$$
\mathrm{T}-\mathrm{SFEHIG}_{\omega, w}\left(\widetilde{P}_{1}, \ldots, \widetilde{P}_{5}\right)=(0.6878,0.4329,0.6591) \text {. }
$$

This shows that the T-SFEIG operator aggregates the membership value.

\section{T-Spherical Fuzzy Einstein Hybrid Interaction Averaging Operators}

In this section, on the basis of new proposed Einstein operations, we define averaging interaction operators in T-spherical fuzzy environment and some basic properties are also discussed.

Definition 7. Consider a collection of T-SFN $P_{j}=\left(m_{j}, i_{j}, n_{j}\right)(j=1,2,3, \ldots, k)$. Then,

$$
\mathrm{T}-\operatorname{SFEWIA}_{w}\left(P_{1}, P_{2}, \ldots, P_{k}\right)=\oplus_{j=1}^{k} w_{j} P_{j}
$$

$$
=\left(\begin{array}{c}
\sqrt[t]{\frac{\prod_{j=1}^{k}\left(1+m_{j}^{t}\right)^{w_{j}}-\prod_{j=1}^{k}\left(1-m_{j}^{t}\right)^{w_{j}}}{\prod_{j=1}^{k}\left(1+m_{j}^{t}\right)^{w_{j}}+\prod_{j=1}^{k}\left(1-m_{j}^{t}\right)^{w_{j}}}, \sqrt[t]{\frac{\prod_{j=1}^{k}\left(1+i_{j}^{t}\right)^{w_{j}}-\prod_{j=1}^{k}\left(1-i_{j}^{t}\right)^{w_{j}}}{\prod_{j=1}^{k}\left(1+i_{j}^{t}\right)^{w_{j}}+\prod_{j=1}^{k}\left(1-i_{j}^{t}\right)^{w_{j}}}},} \\
\sqrt[t]{\frac{2\left(\prod_{j=1}^{k}\left(1-m_{j}^{t}-i_{j}^{t}\right)^{w_{j}}-\prod_{j=1}^{k}\left(1-m_{j}^{t}-i_{j}^{t}-n_{j}^{t}\right)^{w_{j}}\right)}{\prod_{j=1}^{k}\left(1+m_{j}^{t}\right)^{w_{j}}+\prod_{j=1}^{k}\left(1-m_{j}^{t}\right)^{w_{j}}}}
\end{array},\right.
$$

T - SFEWIA ${ }_{w}$ is called T-spherical fuzzy Einstein weighted interactive averaging (T-SFEWIA) operator with weight vector $w=\left(w_{1}, w_{2}, \ldots, w_{k}\right)^{T}$ of $P_{j}$ with $w_{j} \in[0,1]$ and $\sum_{j=1}^{k} w_{j}=1$.
Theorem 7. If all $P_{j}=P_{0}$, then T-SFEWIA ${ }_{w}$ $\left(P_{1}, P_{2}, \ldots, P_{k}\right)=P_{0}$.

Proof. Let $P_{j}=P_{0}=\left(m_{0}, i_{0}, n_{0}\right)$ for all $j$; then, 


$$
\begin{aligned}
& \operatorname{T}-\operatorname{SFEWIA}_{w}\left(P_{1}, P_{2}, \ldots, P_{k}\right)=\left(\begin{array}{c}
\sqrt[t]{\frac{\prod_{j=1}^{k}\left(1+m_{j}^{t}\right)^{w_{j}}-\prod_{j=1}^{k}\left(1-m_{j}^{t}\right)^{w_{j}}}{\prod_{j=1}^{k}\left(1+m_{j}^{t}\right)^{w_{j}}+\prod_{j=1}^{k}\left(1-m_{j}^{t}\right)^{w_{j}}}, \sqrt[t]{\frac{\prod_{j=1}^{k}\left(1+i_{j}^{t}\right)^{w_{j}}-\prod_{j=1}^{k}\left(1-i_{j}^{t}\right)^{w_{j}}}{\prod_{j=1}^{k}\left(1+i_{j}^{t}\right)^{w_{j}}+\prod_{j=1}^{k}\left(1-i_{j}^{t}\right)^{w_{j}}}}} \\
\sqrt[t]{\frac{2\left(\prod_{j=1}^{k}\left(1-m_{j}^{t}-i_{j}^{t}\right)^{w_{j}}-\prod_{j=1}^{k}\left(1-m_{j}^{t}-i_{j}^{t}-n_{j}^{t}\right)^{w_{j}}\right)}{\prod_{j=1}^{k}\left(1+m_{j}^{t}\right)^{w_{j}}+\prod_{j=1}^{k}\left(1-m_{j}^{t}\right)^{w_{j}}}}
\end{array}\right.
\end{aligned}
$$

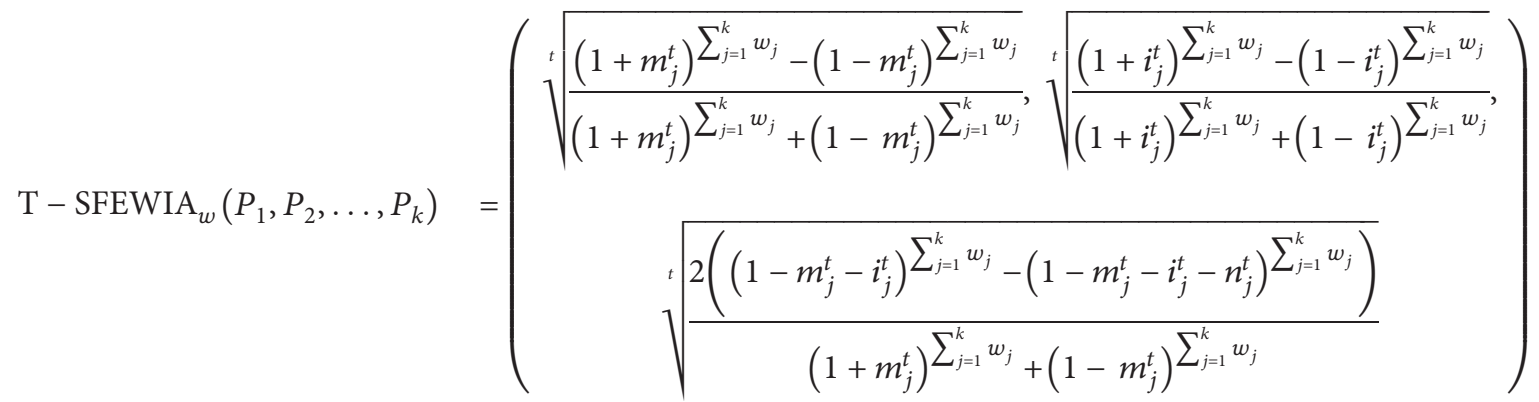

$$
\begin{aligned}
& =\left(m_{0}, i_{0}, n_{0}\right)=P_{0} \text {. }
\end{aligned}
$$

Theorem 8. Consider a collection of T-SFNs $P_{j}(j=1,2, \ldots, k)$ with $P^{L}=\min _{j} P_{j}$ and $P^{U}=\min _{j} P_{j}$. Then,

$$
P^{L} \leq \mathrm{T}-\operatorname{SFEWIA}_{w}\left(P_{1}, P_{2}, \ldots, P_{k}\right) \leq P^{U} .
$$

Proof. Straightforward.
Theorem 9. For any two T-SFNs, $P_{j}=\left(m_{j}, i_{j}, n_{j}\right)$ and $P_{j}^{\prime}=$ $\left(m_{j}^{\prime}, i_{j}^{\prime}, n_{j}^{\prime}\right)$ such that $P_{j} \leq P_{j}^{\prime}$ for all $j$. Then, $\mathrm{T}-\operatorname{SFEWIA}_{w}\left(P_{1}, P_{2}, \ldots, P_{k}\right) \leq \mathrm{T}-\operatorname{SFEWIA}_{w}\left(P_{1}^{\prime}, P_{2}^{\prime}, \ldots, P_{k}^{\prime}\right)$.

Proof. Let $P_{j} \leq P_{j}^{\prime}$; then, $m_{j} \leq m_{j}^{\prime}, i_{j} \leq i_{j}^{\prime}$, and $n_{j} \geq n_{j}^{\prime}$. Then, by using this basic information,

$$
\begin{aligned}
& \sqrt[t]{\frac{2\left(\prod_{j=1}^{k}\left(1-m_{j}^{t}-i_{j}^{t}\right)^{w_{j}}-\prod_{j=1}^{k}\left(1-m_{j}^{t}-i_{j}^{t}-n_{j}^{t}\right)^{w_{j}}\right)}{\prod_{j=1}^{k}\left(1+m_{j}^{t}\right)^{w_{j}}+\prod_{j=1}^{k}\left(1-m_{j}^{t}\right)^{w_{j}}}} \\
& \leq \sqrt[t]{\frac{2\left(\prod_{j=1}^{k}\left(1-\left(m_{j}^{\prime}\right)^{t}-\left(i_{j}^{\prime}\right)^{t}\right)^{w_{j}}-\prod_{j=1}^{k}\left(1-\left(m_{j}^{\prime}\right)^{t}-\left(i_{j}^{\prime}\right)^{t}-\left(n_{j}^{\prime}\right)^{t}\right)^{w_{j}}\right)}{\prod_{j=1}^{k}\left(1+\left(m_{j}^{\prime}\right)^{t}\right)^{w_{j}}+\prod_{j=1}^{k}\left(1-\left(m_{j}^{\prime}\right)^{t}\right)^{w_{j}}}}, \\
& \sqrt[t]{\frac{\prod_{j=1}^{k}\left(1+i_{j}^{t}\right)^{w_{j}}-\prod_{j=1}^{k}\left(1-i_{j}^{t}\right)^{w_{j}}}{\prod_{j=1}^{k}\left(1+i_{j}^{t}\right)^{w_{j}}+\prod_{j=1}^{k}\left(1-i_{j}^{t}\right)^{w_{j}}}} \leq \sqrt[t]{\frac{\prod_{j=1}^{k}\left(1+\left(i_{j}^{\prime}\right)^{t}\right)^{w_{j}}-\prod_{j=1}^{k}\left(1-\left(i_{j}^{\prime}\right)^{t}\right)^{w_{j}}}{\prod_{j=1}^{k}\left(1+\left(i_{j}^{\prime}\right)^{t}\right)^{w_{j}}+\prod_{j=1}^{k}\left(1-\left(i_{j}^{\prime}\right)^{t}\right)^{w_{j}}}},
\end{aligned}
$$

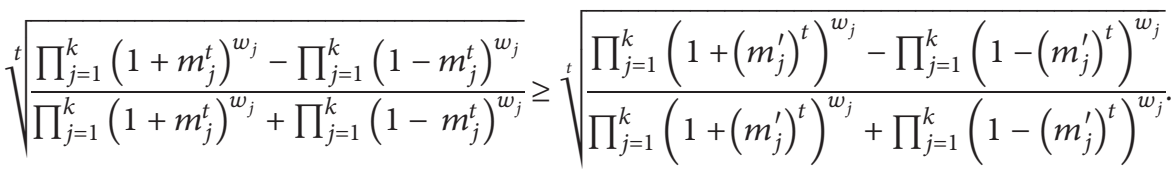


This shows that

$\mathrm{T}-\operatorname{SFEWIA}_{w}\left(P_{1}, P_{2}, \ldots, P_{k}\right) \leq \mathrm{T}-\operatorname{SFEWIA}_{w}\left(P_{1}^{\prime}, P_{2}^{\prime}, \ldots, P_{k}^{\prime}\right)$.
Definition 8. Consider a collection of T-SFNs $P_{j}=\left(m_{j}, i_{j}, n_{j}\right)(j=1,2,3, \ldots, k)$. Then,

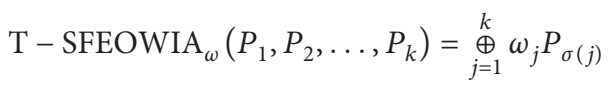

$$
=\left(\frac{\sqrt[t]{\frac{\prod_{j=1}^{k}\left(1+m_{\sigma(j)}^{t}\right)^{\omega_{j}}-\prod_{j=1}^{k}\left(1-m_{\sigma(j)}^{t}\right)^{\omega_{j}}}{\prod_{j=1}^{k}\left(1+m_{\sigma(j)}^{t}\right)^{\omega_{j}}+\prod_{j=1}^{k}\left(1-m_{\sigma(j)}^{t}\right)^{\omega_{j}}}, \sqrt[t]{\frac{\prod_{j=1}^{k}\left(1+i_{\sigma(j)}^{t}\right)^{\omega_{j}}-\prod_{j=1}^{k}\left(1-i_{\sigma(j)}^{t}\right)^{\omega_{j}}}{\prod_{j=1}^{k}\left(1+i_{\sigma(j)}^{t}\right)^{\omega_{j}}+\prod_{j=1}^{k}\left(1-i_{\sigma(j)}^{t}\right)^{\omega_{j}}}}}}{\sqrt[t]{\frac{2\left[\prod_{j=1}^{k}\left(1-m_{\sigma(j)}^{t}-i_{\sigma(j)}^{t}\right)^{\omega_{j}}-\prod_{j=1}^{k}\left(1-m_{\sigma(j)}^{t}-i_{\sigma(j)}^{t}-n_{\sigma(j)}^{t}\right)^{\omega_{j}}\right]}{\prod_{j=1}^{k}\left(1+m_{\sigma(j)}^{t}\right)^{\omega_{j}}+\prod_{j=1}^{k}\left(1-m_{\sigma(j)}^{t}\right)^{\omega_{j}}}}}\right)
$$

$\mathrm{T}-$ SFEOWIA $_{\omega}$ is called the T-spherical fuzzy Einstein ordered weighted interactive averaging (T-SFEOWIA) operator with associated weight vector $\omega=\left(\omega_{1}, \omega_{2}, \ldots, \omega_{k}\right)^{T}$ of $P_{j}$ with $\omega_{j} \in[0,1]$ and $\sum_{j=1}^{k} \omega_{j}=1$. Here, $\underset{\widetilde{P}}{\sigma}(j)$ is any permutation of $(1,2, \ldots, k)$ such that $\widetilde{P}_{\sigma(j-1)} \geq \widetilde{P}_{\sigma(j)}$.

Theorem 10. If for all $P_{j}=P_{0}$, then $T-\operatorname{SFEOWA}_{\omega}\left(P_{1}, P_{2}, \ldots, P_{k}\right)=P_{0}$.

Proof. Same as in Theorem 7.

Theorem 11. Consider a collection of T-SFNs $P_{j}(j=1,2$, $\ldots, k)$ with $P^{L}=\min _{j} P_{j}$ and $P^{U}=\min _{j} P_{j}$. Then,

$$
P^{L} \leq \mathrm{T}-\text { SFEOWIA }_{\omega}\left(P_{1}, P_{2}, \ldots, P_{k}\right) \leq P^{U} .
$$

Proof. Straightforward.

Theorem 12. Consider any two $T$ - SFNs $P_{j}=\left(m_{j}, i_{j}, n_{j}\right)$ and $P_{j}^{\prime}=\left(m_{j}^{\prime}, i_{j}^{\prime}, n_{j}^{\prime}\right)$ such that $P_{j} \leq P_{j}^{\prime}$ for all $j$. Then,

$$
\mathrm{T}-\operatorname{SFEOWIA}_{\omega}\left(P_{1}, P_{2}, \ldots, P_{k}\right) \leq \mathrm{T}-\text { SFEOWIA }_{\omega}
$$

$$
\cdot\left(P_{1}^{\prime}, P_{2}^{\prime}, \ldots, P_{k}^{\prime}\right) \text {. }
$$

Proof. Same as in Theorem 9.

Definition 9. Consider a collection of T-SFNs $P_{j}=\left(m_{j}\right.$, $\left.i_{j}, n_{j}\right)(j=1,2,3, \ldots, k)$. The mapping

$$
\mathrm{T}-\operatorname{SFEHIA}_{w, \omega}\left(\widetilde{P}_{1}, \widetilde{P}_{2}, \ldots, \widetilde{P}_{k}\right)=\oplus_{j=1}^{k} \omega_{j} \widetilde{P}_{\sigma(j)}
$$

$$
=\left(\begin{array}{c}
\sqrt[t]{\frac{\prod_{j=1}^{k}\left(1+\tilde{m}_{\sigma(j)}^{t}\right)^{\omega_{j}}-\prod_{j=1}^{k}\left(1-\tilde{m}_{\sigma(j)}^{t}\right)^{\omega_{j}}}{\prod_{j=1}^{k}\left(1+\tilde{m}_{\sigma(j)}^{t}\right)^{\omega_{j}}+\prod_{j=1}^{k}\left(1-\tilde{m}_{\sigma(j)}^{t}\right)^{\omega_{j}}}, \sqrt[t]{\frac{\prod_{j=1}^{k}\left(1+\widetilde{i}_{\sigma(j)}^{t}\right)^{\omega_{j}}-\prod_{j=1}^{k}\left(1-\widetilde{i}_{\sigma(j)}^{t}\right)^{\omega_{j}}}{\prod_{j=1}^{k}\left(1+\widetilde{i}_{\sigma(j)}^{t}\right)^{\omega_{j}}+\prod_{j=1}^{k}\left(1-\widetilde{i}_{\sigma(j)}^{t}\right)^{\omega_{j}}},}} \\
\sqrt[t]{\frac{2\left(\prod_{j=1}^{k}\left(1-\tilde{m}_{\sigma(j)}^{t}-\widetilde{i}_{\sigma(j)}^{t}\right)^{\omega_{j}}-\prod_{j=1}^{k}\left(1-\widetilde{m}_{\sigma(j)}^{t}-\widetilde{i}_{\sigma(j)}^{t}-n_{\sigma(j)}^{t}\right)^{\omega_{j}}\right)}{\prod_{j=1}^{k}\left(1+\tilde{m}_{\sigma(j)}^{t}\right)^{\omega_{j}}+\prod_{j=1}^{k}\left(1-\tilde{m}_{\sigma(j)}^{t}\right)^{\omega_{j}}}}
\end{array},\right.
$$

is called the T-spherical fuzzy Einstein hybrid interactive averaging (T-SFEHIA) operator, where $\widetilde{P}_{j}=k w_{j} P_{j}$ and $w_{j}, \omega_{j} \in[0,1]$, and $\sum_{j=1}^{k} w_{j}=1$ and $\sum_{j=1}^{k} \omega_{j}=1$.

Hybrid aggregation operators first aggregate the given data considering their attributes and then rearrange them in a specific order. After that, they aggregate the data considering their order. This means that hybrid operators are a generalization of weighted and ordered weighted operators. So, the T-SFEHIA operator will satisfy idempotency, monotonicity, and bounded property. 
Example 2. Consider five T-SFNs $P_{1}=(0.9,0.3,0.4)$, $P_{2}=(0.6,0.3,0.2), P_{3}=(0.3,0.8,0.6), P_{4}=(0.4,0.5,0.8)$, and $P_{5}=(0.6,0.0,0.0)$ with a weight vector $w=(0.25,0.20,0.15,0.18,0.22)^{T}$.

solution 1. First of all, we find the aggregated value of these T-SFNs by using the T-spherical fuzzy Einstein hybrid averaging aggregation (T-SFEHA) operator [42] to find out the drawbacks of given operators. For this purpose, first, we have to find the value of $t$ for which the given data lie in T-spherical fuzzy environment.

$$
\begin{aligned}
& \text { As } 0.3+0.8+0.6=1.7 \text {, } \\
& \text { For } t=2,0.3^{2}+0.8^{2}+0.6^{2}=1.09 \\
& \text { For } t=3,0.3^{3}+0.8^{3}+0.6^{3}=0.755
\end{aligned}
$$

Similarly, for $t=3$, all the given data lie in the T-spherical fuzzy environment.

By using T-spherical fuzzy Einstein weighted averaging operator, we shall be able to find these values:

$$
\begin{aligned}
& P_{1}=(0.9362,0.2104,0.3029), \\
& P_{2}=(0.6,0.3,0.2), \\
& P_{3}=(0.2726,0.8527,0.6984), \\
& P_{4}=(0.3862,0.5437,0.8211), \\
& P_{5}=(0.6187,0.0,0.0) .
\end{aligned}
$$

Their scores values will be

$$
\begin{aligned}
& \operatorname{SC}\left(P_{1}\right)=0.7927 \\
& \operatorname{SC}\left(P_{2}\right)=0.2080 \\
& \operatorname{SC}\left(P_{3}\right)=-0.3203 \\
& \operatorname{SC}\left(P_{4}\right)=-0.4961 \\
& \operatorname{SC}\left(P_{5}\right)=0.2368
\end{aligned}
$$

Now using the score value, the aggregated values obtained by using T-SFEWA operators are rearranged in descending order. Then, these ordered values are again aggregated by using the T-SFEHA operator with associated weight vector being $\omega=(0.112,0.236,0.304,0.236,0.112)$ :

$$
\begin{aligned}
& \widetilde{P}_{\sigma(1)}=(0.9362,0.2104,0.3029), \\
& \widetilde{P}_{\sigma(2)}=(0.6187,0.0,0.0), \\
& \widetilde{P}_{\sigma(3)}=(0.6,0.3,0.2), \\
& \widetilde{P}_{\sigma(4)}=(0.2726,0.8527,0.6984), \\
& \widetilde{P}_{\sigma(5)}=(0.3862,0.5437,0.8211) .
\end{aligned}
$$

Now, employ the T-SFEHA operator as follows:

$$
\mathrm{T}-\operatorname{SFEHA}_{w, \omega}\left(\widetilde{P}_{1}, \ldots, \widetilde{P}_{5}\right)=(0.6187,0.0,0.0) \text {. }
$$

From the above result, it is noticed that when abstinence or nonmembership value of one T-SFN is zero, then the T-SFEHA operator cannot aggregate the whole abstinence and nonmembership value. This shows a big flaw in the T-SFEHA operator. This means that the results obtained from T-SFEHA operators are not reliable. Now, by using the T-SFEIA operator, we shall show that the proposed operator will overcome this drawback.

By using T-SFEIA operator, we shall be able to find

$$
\begin{aligned}
& P_{1}=(0.9362,0.4308,0.2739), \\
& P_{2}=(0.6,0.3,0.2), \\
& P_{3}=(0.2726,0.7370,0.5956), \\
& P_{4}=(0.3862,0.4829,0.7888), \\
& P_{5}=(0.6187,0.0,0.0) .
\end{aligned}
$$

Their scores values will be

$$
\begin{aligned}
& \operatorname{SC}\left(P_{1}\right)=0.7999 \\
& \operatorname{SC}\left(P_{2}\right)=0.2080 \\
& \operatorname{SC}\left(P_{3}\right)=-0.1910 \\
& \operatorname{SC}\left(P_{4}\right)=-0.4333 \\
& \operatorname{SC}\left(P_{5}\right)=0.2368
\end{aligned}
$$

Now using the score value, the aggregated values obtained by using T-SFEWIA operators are rearranged in descending order. Then, these ordered values are again aggregated by using the T-SFEHIA operator, with associated weight vector being $\omega=(0.112,0.236,0.304,0.236,0.112)$ :

$$
\begin{aligned}
& \widetilde{P}_{\sigma(1)}=(0.9362,0.4308,0.2739), \\
& \widetilde{P}_{\sigma(2)}=(0.6187,0.0,0.0), \\
& \widetilde{P}_{\sigma(3)}=(0.6,0.3,0.2), \\
& \widetilde{P}_{\sigma(4)}=(0.2726,0.7370,0.5956), \\
& \widetilde{P}_{\sigma(5)}=(0.3862,0.4829,0.7888) .
\end{aligned}
$$

Now, employ the T-SFEHIA operator as follows:

$$
\mathrm{T}-\operatorname{SFEHIA}_{w, \omega}\left(\widetilde{P}_{1}, \ldots, \widetilde{P}_{5}\right)=(0.6372,0.5055,0.3978) \text {. }
$$

This shows that the T-SFEIA operator aggregates the membership value.

\section{Advantages}

In this section, we prove that our work is more generalized than the existing work. In our proposed work, experts are free in giving the values to alternatives according to the given attributes. Not only this, the proposed work is also valid under those conditions where the existing work fails. Here, we reduced the proposed work to intuitionistic, Pythagorean, q-rung orthopair, picture, and spherical fuzzy environments. This proves that the proposed work is valid for all those environments.

Consider the T-SFEHIA defined as follows:

(1) For $t=2$, equation (31) reduces to SF Einstein hybrid interaction averaging operators (SFEHIA operators), i.e., 


$$
\operatorname{T-SFEHIA} A_{w, \omega}\left(\widetilde{P}_{1}, \widetilde{P}_{2}, \ldots, \widetilde{P}_{k}\right)=\left(\begin{array}{c}
\sqrt{\frac{\prod_{j=1}^{k}\left(1+\widetilde{m}_{\sigma(j)}^{2}\right)^{\omega_{j}}-\prod_{j=1}^{k}\left(1-\widetilde{m}_{\sigma(j)}^{2}\right)^{\omega_{j}}}{\prod_{j=1}^{k}\left(1+\widetilde{m}_{\sigma(j)}^{2}\right)^{\omega_{j}}+\prod_{j=1}^{k}\left(1-\widetilde{m}_{\sigma(j)}^{2}\right)^{\omega_{j}}}, \sqrt{\frac{\prod_{j=1}^{k}\left(1+\widetilde{i}_{\sigma(j)}^{2}\right)^{\omega_{j}}-\prod_{j=1}^{k}\left(1-\widetilde{i}_{\sigma(j)}^{2}\right)^{\omega_{j}}}{\prod_{j=1}^{k}\left(1+\widetilde{i}_{\sigma(j)}^{2}\right)^{\omega_{j}}+\prod_{j=1}^{k}\left(1-\widetilde{i}_{\sigma(j)}^{2}\right)^{\omega_{j}}}}} \\
\sqrt{\frac{2\left(\prod_{j=1}^{k}\left(1-\tilde{m}_{\sigma(j)}^{2}-\widetilde{i}_{\sigma(j)}^{2}\right)^{\omega_{j}}-\prod_{j=1}^{k}\left(1-\widetilde{m}_{\sigma(j)}^{2}-\widetilde{i}_{\sigma(j)}^{2}-n_{\sigma(j)}^{t}\right)_{2}^{\omega_{j}}\right)}{\prod_{j=1}^{k}\left(1+\widetilde{m}_{\sigma(j)}^{2}\right)^{\omega_{j}}+\prod_{j=1}^{k}\left(1-\widetilde{m}_{\sigma(j)}^{2}\right)^{\omega_{j}}}}
\end{array} .\right.
$$

(2) For $t=1$, equation (31) reduces to PF Einstein hybrid interaction averaging operators (PFEHIA operators), i.e.,

$$
\operatorname{PFEHA}_{w, \omega}\left(\widetilde{P}_{1}, \widetilde{P}_{2}, \ldots, \widetilde{P}_{k}\right)=\left(\begin{array}{c}
\frac{\prod_{j=1}^{k}\left(1+\widetilde{m}_{\sigma(j)}\right)^{\omega_{j}}-\prod_{j=1}^{k}\left(1-\widetilde{m}_{\sigma(j)}\right)^{\omega_{j}}}{\prod_{j=1}^{k}\left(1+\widetilde{m}_{\sigma(j)}\right)^{\omega_{j}}+\prod_{j=1}^{k}\left(1-\widetilde{m}_{\sigma(j)}\right)^{\omega_{j}},} \frac{\prod_{j=1}^{k}\left(1+\widetilde{i}_{\sigma(j)}\right)^{\omega_{j}}-\prod_{j=1}^{k}\left(1+\widetilde{i}_{\sigma(j)}\right)^{\omega_{j}}+\prod_{j=1}^{k}\left(1-\widetilde{i}_{\sigma(j)}\right)^{\omega_{j}}}{\left.\prod_{\sigma(j)}\right)^{\omega_{j}}} \\
\frac{2\left(\prod_{j=1}^{k}\left(1-\widetilde{m}_{\sigma(j)}\right)^{\omega_{j}}-\prod_{j=1}^{k}\left(1-\widetilde{m}_{\sigma(j)}-\widetilde{i}_{\sigma(j)} n_{\sigma(j)}\right)^{\omega_{j}}\right)}{\prod_{j=1}^{k}\left(1+\widetilde{m}_{\sigma(j)}\right)^{\omega_{j}}+\prod_{j=1}^{k}\left(1-\widetilde{m}_{\sigma(j)}\right)^{\omega_{j}}}
\end{array}\right) .
$$

(3) For $i=0$, equation (31) reduces to q-ROPF Einstein hybrid interaction averaging operators (q-ROFEHIA operators), i.e.,

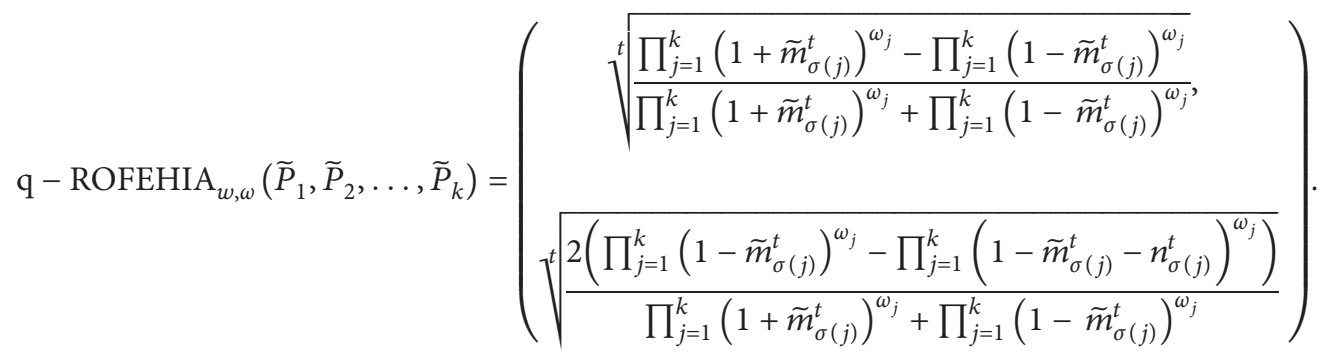

(4) For $t=2$ and $i=0$, equation (31) reduces to PyF Einstein hybrid interaction averaging operators (PyFEHIA operators), i.e.,

$$
\operatorname{PyFEHIA}_{w, \omega}\left(\widetilde{P}_{1}, \widetilde{P}_{2}, \ldots, \widetilde{P}_{k}\right)\left(\begin{array}{c}
\sqrt{\frac{\prod_{j=1}^{k}\left(1+\widetilde{m}_{\sigma(j)}^{2}\right)^{\omega_{j}}-\prod_{j=1}^{k}\left(1-\widetilde{m}_{\sigma(j)}^{2}\right)^{\omega_{j}}}{\prod_{j=1}^{k}\left(1+\widetilde{m}_{\sigma(j)}^{2}\right)^{\omega_{j}}+\prod_{j=1}^{k}\left(1-\widetilde{m}_{\sigma(j)}^{2}\right)^{\omega_{j}}}} \\
\sqrt{\frac{2\left(\prod_{j=1}^{k}\left(1-\widetilde{m}_{\sigma(j)}^{2}\right)^{\omega_{j}}-\prod_{j=1}^{k}\left(1-\widetilde{m}_{\sigma(j)}^{2}-\widetilde{n}_{\sigma(j)}^{2}\right)^{\omega_{j}}\right)}{\prod_{j=1}^{k}\left(1+\widetilde{m}_{\sigma(j)}^{2}\right)^{\omega_{j}}+\prod_{j=1}^{k}\left(1-\widetilde{m}_{\sigma(j)}^{2}\right)^{\omega_{j}}}}
\end{array} .\right.
$$


(5) For $t=1$ and $i=0$, equation (31) reduces to IF Einstein hybrid interaction averaging operators (IFEHA operators), i.e.,

$$
\operatorname{IFEHIA}_{w, \omega}\left(\widetilde{P}_{1}, \widetilde{P}_{2}, \ldots, \widetilde{P}_{k}\right)=\left(\begin{array}{c}
\frac{\left.\prod_{j=1}^{k}\left(1+\widetilde{m}_{\sigma(j)}\right)\right)^{\omega_{j}}-\prod_{j=1}^{k}\left(1-\widetilde{m}_{\sigma(j)}\right)^{\omega_{j}}}{\prod_{j=1}^{k}\left(1+\widetilde{m}_{\sigma(j)}\right)^{\omega_{j}}+\prod_{j=1}^{k}\left(1-\widetilde{m}_{\sigma(j)}\right)^{\omega_{j}}}, \\
\frac{2\left(\prod_{j=1}^{k}\left(1-\widetilde{m}_{\sigma(j)}\right)^{\omega_{j}}-\prod_{j=1}^{k}\left(1-\widetilde{m}_{\sigma(j)}-\widetilde{n}_{\sigma(j)}\right)^{\omega_{j}}\right)}{\prod_{j=1}^{k}\left(1+\widetilde{m}_{\sigma(j)}\right)^{\omega_{j}}+\prod_{j=1}^{k}\left(1-\widetilde{m}_{\sigma(j)}\right)^{\omega_{j}}}
\end{array}\right)
$$

Similarly, we can reduce T-SFEWIA operator, T-SFEOWIA operator, T-SFEWIG operator, T-SFEOWIG operator, and T-SFEHIG operator.

The proposed aggregation operators can aggregate the data given in FS, IFS, PyFS, q-ROPFS, PFS, and spherical fuzzy set environments, but the converse is not possible. Here, with the help of an example, it is proved that the proposed aggregation operator can aggregate the data given in IFSs.

Example 4. Let IFNs $P_{1}=(0.2,0.5), P_{2}=(0.7,0.1)$, $P_{3}=(0.3,0.4), P_{4}=(0.6,0.2)$, and $P_{5}=(0.5,0.5)$ with a weight vector $w=(0.25,0.20,0.15,0.18,0.22)^{T}$.

Solution 1. We can write these IFNs in the form of T-SFNs as $P_{1}=(0.2,0,0.5), \quad P_{2}=(0.7,0,0.1), \quad P_{3}=(0.3,0,0.4)$, $P_{4}=(0.6,0,0.2)$, and $P_{5}=(0.5,0,0.5)$. Then, by using the T-SFEWIA operator, we shall be able to find these values:

$$
\begin{aligned}
& P_{1}=(0.2481,0,0.5312), \\
& P_{2}=(0.7,0,0.1), \\
& P_{3}=(0.2281,0,0.3630), \\
& P_{4}=(0.5538,0,0.2071), \\
& P_{5}=(0.5401,0,0.4599) .
\end{aligned}
$$

Their scores values will be

$$
\begin{aligned}
& \operatorname{SC}\left(P_{1}\right)=-0.2831, \\
& \operatorname{SC}\left(P_{2}\right)=0.6 \\
& \operatorname{SC}\left(P_{3}\right)=-0.1350 \\
& \operatorname{SC}\left(P_{4}\right)=0.3467 \\
& \operatorname{SC}\left(P_{5}\right)=0.0801
\end{aligned}
$$

Now, using the score value, the aggregated values obtained by using T-SFEWIA operators are rearranged in descending order. Then, these ordered values are again aggregated by using the T-SFEHIA operator with the associated weight vector being $\omega=(0.112,0.236$, $0.304,0.236,0.112)$ :

$$
\begin{aligned}
& \widetilde{P}_{\sigma(1)}=(0.7,0,0.1), \\
& \widetilde{P}_{\sigma(2)}=(0.5538,0,0.2071), \\
& \widetilde{P}_{\sigma(3)}=(0.5401,0,0.4599), \\
& \widetilde{P}_{\sigma(4)}=(0.2281,0,0.3630), \\
& \widetilde{P}_{\sigma(5)}=(0.2481,0,0.5312) .
\end{aligned}
$$

Now, utilize the T-SFEHIA operator as follows:

$$
\mathrm{T}-\operatorname{SFEHIA}_{w, \omega}\left(\widetilde{P}_{1}, \widetilde{P}_{2}, \ldots, \widetilde{P}_{5}\right)=(0.4709,0,0.2645) .
$$

Here, it is proved that the information given in IFNs can be solved by using the T-SFEHIA operator. Similarly, we can solve the information given in IFNs by using the T-SEHIG operator and the information given in any other fuzzy structure can also be aggregated using the proposed operators.

\section{An Algorithm for MADM with T-Spherical Fuzzy Information}

Consider a set of alternatives $D=\left\{d_{1}, d_{2}, d_{3}, \ldots, d_{l}\right\}$ and a set of attributes $M=\left\{m_{1}, m_{2}, m_{3}, \ldots, m_{k}\right\}$ having a weight vector $w=\left\{w_{1}, w_{2}, w_{3}, \ldots, w_{l}\right\}$, where $w_{j} \in[0,1]$ and $\sum_{m=1}^{l}$ $w_{m}=1$. For making a decision, we have to follow these steps:

Step 1. Calculate $t$ for which the values lie in T-spherical information

Step 2. Aggregate the given alternatives according to attributes by T-SFEWIA (or T-SFEWIG) operators using some weight vectors

Step 3. Find scores values and with the help of score value, we reorder them in descending order

Step 4. Aggregate these ordered values using T-SFEHIA (or T-SFEHIG) operator

Step 5. Using score values find out the best option

Example 3. The board of governors of a company decided to reduce their expenses for maximizing the profit. They observe that the cost of electricity is one of the major expense and they can reduce it if they started to generate electricity using solar energy. They have three options of photovoltaic cells that they may use in their solar plant: 
TABLE 1: Decision matrix.

\begin{tabular}{lccccc}
\hline & $M_{1}$ & $M_{2}$ & $M_{3}$ & $M_{4}$ & $M_{5}$ \\
\hline$d_{1}$ & $(0.4,0.1,0.7)$ & $(0.5,0.2,0.4)$ & $(0.8,0.3,0.7)$ & $(0.4,0.8,0.5)$ & $(0.9,0.5,0.2)$ \\
$d_{2}$ & $(0.7,0.4,0.3)$ & $(0.2,0.4,0.7)$ & $(0.9,0.3,0.6)$ & $(0.3,0.2,0.8)$ & $(0.4,0.7,0.5)$ \\
$d_{3}$ & $(0.4,0.7,0.5)$ & $(0.6,0.6,0.1)$ & $(0.6,0.9,0.2)$ & $(0.8,0.1,0.1)$ & $(0.5,0.6,0.2)$ \\
\hline
\end{tabular}

TABLE 2: Aggregated values.

\begin{tabular}{|c|c|c|c|c|c|}
\hline & $M_{1}$ & $M_{2}$ & $M_{3}$ & $M_{4}$ & $M_{5}$ \\
\hline$d_{1}$ & $\left(\begin{array}{c}0.4308, \\
0.1077, \\
0.7367\end{array}\right)$ & $\left(\begin{array}{l}0.5, \\
0.2 \\
0.4\end{array}\right)$ & $\left(\begin{array}{c}0.7370, \\
0.2726, \\
0.7172\end{array}\right)$ & $\left(\begin{array}{c}0.3862, \\
0.7770, \\
0.5022\end{array}\right)$ & $\left(\begin{array}{c}0.9164, \\
0.5160, \\
0.1906\end{array}\right)$ \\
\hline$d_{2}$ & $\left(\begin{array}{c}0.4308, \\
0.4308, \\
0.3061\end{array}\right)$ & $\left(\begin{array}{c}0.2, \\
0.4 \\
0.7\end{array}\right)$ & $\left(\begin{array}{c}0.8440, \\
0.2726, \\
0.6677\end{array}\right)$ & $\left(\begin{array}{c}0.2896, \\
0.1931, \\
0.7820\end{array}\right)$ & $\left(\begin{array}{l}0.4129 \\
0.7206 \\
0.5037\end{array}\right)$ \\
\hline$d_{3}$ & $\left(\begin{array}{c}0.4308, \\
0.7485, \\
0.5064\end{array}\right)$ & $\left(\begin{array}{l}0.6, \\
0.6 \\
0.1\end{array}\right)$ & $\left(\begin{array}{c}0.5464, \\
0.8440, \\
0.2430\end{array}\right)$ & $\left(\begin{array}{c}0.7770, \\
0.0965, \\
0.0829\end{array}\right)$ & $\left(\begin{array}{c}0.5160, \\
0.6187 \\
0.2031\end{array}\right)$ \\
\hline
\end{tabular}

(i) $d_{1}$ : monocrystalline photovoltaic cell

(ii) $d_{2}$ : polycrystalline photovoltaic cell

(iii) $d_{3}$ : thin Film photovoltaic cell

They assess the given photovoltaic cell on the basis of the following attributes.

(i) $M_{1}$ : heat tolerance

(ii) $M_{2}:$ cost

(iii) $M_{3}$ : reliability

(iv) $M_{4}$ : efficiency

(v) $M_{5}$ : ability of charge separation

Step 1. As $0.8+0.3+0.7=1.8 \notin[0,1], 0.8^{2}+0.3^{2}+$ $0.7^{2}=1.22 \notin[0,1]$ and $0.8^{3}+0.3^{3}+0.7^{3}=0.882 \epsilon$ $[0,1]$. Similarly, we found that all values in Table 1 belong to $[0,1]$ for $t=3$.

Step 2. By taking $w=(0.25,0.20,0.15,0.18,0.22)^{T}$, we find T-SFEWIA values of given data, as listed in Table 2.

Step 3. Scores of each alternative with respect to all attributes are shown in Table 3.

By comparing the score values, we have

$$
\begin{aligned}
& \operatorname{SC}\left(P_{15}\right)>\operatorname{SC}\left(P_{12}\right)>\operatorname{SC}\left(P_{13}\right)>\operatorname{SC}\left(P_{14}\right)>\operatorname{SC}\left(P_{11}\right) \\
& \operatorname{SC}\left(P_{21}\right)>\operatorname{SC}\left(P_{23}\right)>\operatorname{SC}\left(P_{25}\right)>\operatorname{SC}\left(P_{22}\right)>\operatorname{SC}\left(P_{24}\right) \\
& \operatorname{SC}\left(P_{34}\right)>\operatorname{SC}\left(P_{32}\right)>\operatorname{SC}\left(P_{33}\right)>\operatorname{SC}\left(P_{35}\right)>\operatorname{SC}\left(P_{31}\right) .
\end{aligned}
$$

Based on above score analysis, the data are arranged in descending order and the aggregated values of ordered data are as listed in Table 4.
Step 4. The associated weight vector will be $\omega=(0.112,0.236,0.304,0.236,0.112)^{T}$, and by using T-SFEHA operators, we have

$$
\begin{aligned}
& \widetilde{P}_{1}=(0.6596,0.5227,0.4668), \\
& \widetilde{P}_{2}=(0.6176,0.5291,0.5276), \\
& \widetilde{P}_{3}=(0.5826,0.7075,0.2290) .
\end{aligned}
$$

Step 5. Now, we have to find the score values:

$$
\begin{aligned}
& \operatorname{SC}\left(\widetilde{P}_{1}\right)=0.1853 \\
& \operatorname{SC}\left(\widetilde{P}_{2}\right)=0.0887 \\
& \operatorname{SC}\left(\widetilde{P}_{3}\right)=0.1858 \\
& \operatorname{SC}\left(\widetilde{P}_{3}\right)>\operatorname{SC}\left(\widetilde{P}_{1}\right)>\operatorname{SC}\left(\widetilde{P}_{2}\right) .
\end{aligned}
$$

Since the score value of $d_{3}$ is highest, the thin film photovoltaic cell is the best option.

Now, we check their validity by using Einstein hybrid geometric interaction operators.

By taking $w=(0.25,0.20,0.15,0.18,0.22)^{T}$, we find T-SFEWG values of given data, as listed in Table 5.

Scores of each alternative with respect to all attributes are shown in Table 6.

By comparing the score values, we have

$$
\begin{aligned}
& \operatorname{SC}\left(P_{15}\right)>\operatorname{SC}\left(P_{13}\right)>\operatorname{SC}\left(P_{12}\right)>\operatorname{SC}\left(P_{14}\right)>\operatorname{SC}\left(P_{11}\right), \\
& \operatorname{SC}\left(P_{23}\right)>\operatorname{SC}\left(P_{21}\right)>\operatorname{SC}\left(P_{25}\right)>\operatorname{SC}\left(P_{22}\right)>\operatorname{SC}\left(P_{24}\right), \\
& \operatorname{SC}\left(P_{34}\right)>\operatorname{SC}\left(P_{33}\right)>\operatorname{SC}\left(P_{32}\right)>\operatorname{SC}\left(P_{35}\right)>\operatorname{SC}\left(P_{31}\right) .
\end{aligned}
$$


TABLE 3: Score values.

\begin{tabular}{lccccc}
\hline & $M_{1}$ & $M_{2}$ & $M_{3}$ & $M_{4}$ & $M_{5}$ \\
\hline$d_{1}$ & -0.3199 & 0.0610 & 0.0314 & -0.0690 & 0.7626 \\
$d_{2}$ & 0.3906 & -0.3350 & 0.3035 & -0.4538 & -0.0574 \\
$d_{3}$ & -0.0499 & 0.2150 & 0.1488 & 0.4685 & 0.1290 \\
\hline
\end{tabular}

TABLE 4: Ordered aggregated values.

\begin{tabular}{|c|c|c|c|c|c|}
\hline & $M_{1}$ & $M_{2}$ & $M_{3}$ & $M_{4}$ & $M_{5}$ \\
\hline$d_{1}$ & $\left(\begin{array}{c}0.9164, \\
0.5160, \\
0.1906\end{array}\right)$ & $\left(\begin{array}{c}0.5, \\
0.2, \\
0.4\end{array}\right)$ & $\left(\begin{array}{c}0.7370, \\
0.2726, \\
0.7172\end{array}\right)$ & $\left(\begin{array}{c}0.3862, \\
0.7770, \\
0.5022\end{array}\right)$ & $\left(\begin{array}{c}0.4308, \\
0.1077 \\
0.7367\end{array}\right)$ \\
\hline$d_{2}$ & $\left(\begin{array}{c}0.7485, \\
0.4308, \\
0.3061\end{array}\right)$ & $\left(\begin{array}{c}0.8440 \\
02726 \\
0.6677\end{array}\right)$ & $\left(\begin{array}{c}0.4129, \\
0.7206, \\
0.5037\end{array}\right)$ & $\left(\begin{array}{c}0.2, \\
0.4, \\
0.7\end{array}\right)$ & $\left(\begin{array}{c}0.2896, \\
0.1931 \\
0.7820\end{array}\right)$ \\
\hline$d_{3}$ & $\left(\begin{array}{c}0.7770, \\
0.0965, \\
0.0829\end{array}\right)$ & $\left(\begin{array}{c}0.6, \\
0.6, \\
0.1\end{array}\right)$ & $\left(\begin{array}{c}0.5464, \\
0.8440, \\
0.2430\end{array}\right)$ & $\left(\begin{array}{c}0.5160, \\
0.6187, \\
0.2031\end{array}\right)$ & $\left(\begin{array}{c}0.4308, \\
0.7485, \\
0.5064\end{array}\right)$ \\
\hline
\end{tabular}

Table 5: Aggregated values.

\begin{tabular}{|c|c|c|c|c|c|}
\hline & $M_{1}$ & $M_{2}$ & $M_{3}$ & $M_{4}$ & $M_{5}$ \\
\hline$d_{1}$ & $\left(\begin{array}{c}0.4117, \\
0.1077, \\
0.7485\end{array}\right)$ & $\left(\begin{array}{l}0.5, \\
0.2, \\
0.4\end{array}\right)$ & $\left(\begin{array}{c}0.7998, \\
0.2726, \\
0.6398\end{array}\right)$ & $\left(\begin{array}{c}0.4008, \\
0.7770, \\
0.4829\end{array}\right)$ & $\left(\begin{array}{c}0.9051, \\
0.5160, \\
0.2064\end{array}\right)$ \\
\hline$d_{2}$ & $\left(\begin{array}{c}0.7347, \\
0.4308, \\
0.3232\end{array}\right)$ & $\left(\begin{array}{l}0.2, \\
0.4, \\
0.7\end{array}\right)$ & $\left(\begin{array}{c}0.9071, \\
0.2726, \\
0.5464\end{array}\right)$ & $\left(\begin{array}{c}0.2984, \\
0.1931, \\
0.7770\end{array}\right)$ & $\left(\begin{array}{c}0.4034, \\
0.7206, \\
0.5160\end{array}\right)$ \\
\hline$d_{3}$ & $\left(\begin{array}{c}0.4064, \\
0.7485, \\
0.5381\end{array}\right)$ & $\left(\begin{array}{l}0.6, \\
0.6, \\
0.1\end{array}\right)$ & $\left(\begin{array}{c}0.6434, \\
0.8440, \\
0.1817\end{array}\right)$ & $\left(\begin{array}{c}0.7807, \\
0.0965, \\
0.0965\end{array}\right)$ & $\left(\begin{array}{c}0.5103, \\
0.6187, \\
0.2064\end{array}\right)$ \\
\hline
\end{tabular}

TABLE 6: Score values.

\begin{tabular}{lccccc}
\hline & $M_{1}$ & $M_{2}$ & $M_{3}$ & $M_{4}$ & $M_{5}$ \\
\hline$d_{1}$ & -0.3495 & 0.0610 & 0.2497 & -0.0482 & 0.7327 \\
$d_{2}$ & 0.3628 & -0.3350 & 0.5833 & -0.4425 & -0.0717 \\
$d_{3}$ & -0.0887 & 0.2150 & 0.2603 & 0.4749 & 0.1241 \\
\hline
\end{tabular}

TABLE 7: Ordered aggregated values.

\begin{tabular}{|c|c|c|c|c|c|}
\hline & $M_{1}$ & $M_{2}$ & $M_{3}$ & $M_{4}$ & $M_{5}$ \\
\hline$d_{1}$ & $\left(\begin{array}{c}0.9051, \\
0.5160, \\
0.2064\end{array}\right)$ & $\left(\begin{array}{c}0.7998, \\
0.2726, \\
0.6398\end{array}\right)$ & $\left(\begin{array}{c}0.5, \\
0.2, \\
0.4\end{array}\right)$ & $\left(\begin{array}{c}0.4008, \\
0.7770, \\
0.4829\end{array}\right)$ & $\left(\begin{array}{c}0.4117 \\
0.1077 \\
0.7485\end{array}\right)$ \\
\hline$d_{2}$ & $\left(\begin{array}{c}0.9071, \\
0.2726, \\
0.5464\end{array}\right)$ & $\left(\begin{array}{l}0.7347, \\
0.4308, \\
0.3232\end{array}\right)$ & $\left(\begin{array}{c}0.4034 \\
0.7206 \\
0.5160\end{array}\right)$ & $\left(\begin{array}{c}0.2, \\
0.4, \\
0.7\end{array}\right)$ & $\left(\begin{array}{c}0.2984, \\
0.1931, \\
0.7770\end{array}\right)$ \\
\hline$d_{3}$ & $\left(\begin{array}{c}0.7807, \\
0.0965 \\
0.0965\end{array}\right)$ & $\left(\begin{array}{c}0.6434, \\
0.8440, \\
0.1817\end{array}\right)$ & $\left(\begin{array}{c}0.6, \\
0.6, \\
0.1\end{array}\right)$ & $\left(\begin{array}{l}0.5103, \\
0.6187 \\
0.2064\end{array}\right)$ & $\left(\begin{array}{c}0.4064, \\
0.7485, \\
0.5381\end{array}\right)$ \\
\hline
\end{tabular}


Based on the above score analysis, the data are arranged in descending order and the aggregated values of ordered data are as listed in Table 7.

Associated weight vector will be $\omega=(0.112$, $0.236,0.304,0.236,0.112)^{T}$, and by using T-SFEHG operators, we have

$$
\begin{aligned}
& \widetilde{P}_{1}=(0.5445,0.5217,0.5419), \\
& \widetilde{P}_{2}=(0.6830,0.5376,0.5913), \\
& \widetilde{P}_{3}=(0.7556,0.6879,0.2780) .
\end{aligned}
$$

Step 6. Now, we have to find the score values:

$$
\begin{aligned}
& \operatorname{SC}\left(\widetilde{P}_{1}\right)=0.0023, \\
& \operatorname{SC}\left(\widetilde{P}_{2}\right)=0.1080, \\
& \operatorname{SC}\left(\widetilde{P}_{3}\right)=0.4099, \\
& \operatorname{SC}\left(\widetilde{P}_{3}\right)>\operatorname{SC}\left(\widetilde{P}_{2}\right)>\operatorname{SC}\left(\widetilde{P}_{1}\right) .
\end{aligned}
$$

Here again, the score value of alternative $d_{3}$ is high. So, the thin film photovoltaic cell is the best option.

\section{Conclusion}

In this paper, it is pointed out that the existing work $[14,42]$ fails under some conditions such as in Einstein averaging operators, if one nonmembership value of an IFN is zero, then the NMD of aggregated value will also become zero and neglect the other nonmembership values. Similarly, if the membership value of one IFN becomes zero, then the MD of the aggregated value of the Einstein geometric operator will also become zero and neglect the other membership values. So, new interactive operational laws are proposed in this article. On the basis of these operational laws, T-spherical Einstein interactive geometric operators and T-spherical Einstein interactive averaging operators are proposed. After that, some conditions are discussed under which the proposed operators can reduce to other fuzzy frameworks. A comparison of proposed and existing work is also established and explained using an example. We validate the proposed operators with the help of an application in MADM. In the future, we have a plan to propose some power aggregation operations for T-spherical fuzzy sets and try to use them in the MADM process.

\section{Data Availability}

The data used in this article are artificial and hypothetical, and anyone can use these data before prior permission by just citing this article.

\section{Conflicts of Interest}

The authors declare that they have no conflicts of interest.

\section{Acknowledgments}

This paper was supported by Major Humanities and Social Sciences Research Projects in Zhejiang Universities (no. 2018QN058), China Postdoctoral Science Foundation (no. 2019M651403), Zhejiang Province Natural Science Foundation (no. LY18G010007), and Ningbo Province Natural Science Foundation (no. 2019A610037) and the Fundamental Research Funds for the Provincial Universities of Zhejiang.

\section{References}

[1] L. A. Zadeh, "Fuzzy sets," Information and Control, vol. 8, no. 3, pp. 338-353, 1965.

[2] K. T. Atanassov, "Intuitionistic fuzzy sets," Fuzzy Sets and Systems, vol. 20, no. 1, pp. 87-96, 1986.

[3] K. T. Atanassov, Intuitionistic Fuzzy Sets, Springer-Verlag, Heidelberg, Germany, 1999.

[4] R. R. Yager, "Pythagorean fuzzy subsets," in Proceedings of the 2013 Joint IFSA World Congress and NAFIPS Annual Meeting (IFSA/NAFIPS), Edmonton, AB, Canada, June 2013.

[5] R. R. Yager, "Pythagorean membership grades in multicriteria decision making," IEEE Transactions on Fuzzy Systems, vol. 22, no. 4, pp. 958-965, 2014.

[6] R. R. Yager, "Generalized orthopair fuzzy sets," IEEE Transactions on Fuzzy Systems, vol. 25, no. 5, pp. 1222-1230, 2017.

[7] B. C. Cường, "Picture fuzzy sets," Journal of Computer Science and Cybernetics, vol. 30, no. 4, p. 409, 2015.

[8] T. Mahmood, K. Ullah, Q. Khan, and N. Jan, "An approach toward decision-making and medical diagnosis problems using the concept of spherical fuzzy sets," Neural Computing and Applications, vol. 31, no. 11, pp. 7041-7053, 2018.

[9] Z. Xu and R. R. Yager, "Some geometric aggregation operators based on intuitionistic fuzzy sets," International Journal of General Systems, vol. 35, no. 4, pp. 417-433, 2006.

[10] S. Zeng, W. Su, and C. Zhang, "Intuitionistic fuzzy generalized probabilistic ordered weighted averaging operator and its application to group decision making," Technological and Economic Development of Economy, vol. 22, no. 2, pp. 177193, 2015

[11] H. Garg, "Some robust improved geometric aggregation operators under interval-valued intuitionistic fuzzy environment for multi-criteria decision-making process," Journal of Industrial \& Management Optimization, vol. 14, no. 1, pp. 283-308, 2018.

[12] C. Zhang, C. Chen, D. Streimikiene, and T. Balezentis, "Intuitionistic fuzzy MULTIMOORA approach for multicriteria assessment of the energy storage technologies," Applied Soft Computing, vol. 79, pp. 410-423, 2019.

[13] Y. He, H. Chen, L. Zhou, J. Liu, and Z. Tao, "Intuitionistic fuzzy geometric interaction averaging operators and their application to multi-criteria decision making," Information Sciences, vol. 259, pp. 142-159, 2014.

[14] X. Zhao and G. Wei, "Some intuitionistic fuzzy Einstein hybrid aggregation operators and their application to multiple attribute decision making," Knowledge-Based Systems, vol. 37, pp. $472-479,2013$.

[15] Z. Mu, S. Zeng, and Q. Liu, "Some interval-valued intuitionistic fuzzy Zhenyuan aggregation operators and their application to multi-attribute decision making," International 
Journal of Uncertainty, Fuzziness and Knowledge-Based Systems, vol. 26, no. 4, pp. 633-653, 2018.

[16] S. Zeng, S.-M. Chen, and K.-Y. Fan, "Interval-valued intuitionistic fuzzy multiple attribute decision making based on nonlinear programming methodology and TOPSIS method," Information Sciences, vol. 506, pp. 424-442, 2020.

[17] S. Zeng, S.-M. Chen, and L.-W. Kuo, "Multiattribute decision making based on novel score function of intuitionistic fuzzy values and modified VIKOR method," Information Sciences, vol. 488, pp. 76-92, 2019.

[18] P. Wang and P. Liu, "Some Maclaurin symmetric mean aggregation operators based on Schweizer-Sklar operations for intuitionistic fuzzy numbers and their application to decision making," Journal of Intelligent \& Fuzzy Systems, vol. 36, no. 4, pp. 3801-3824, 2019.

[19] H. Garg, "A new generalized Pythagorean fuzzy information aggregation using Einstein operations and its application to decision making," International Journal of Intelligent Systems, vol. 31, no. 9, pp. 886-920, 2016.

[20] G. Wei and M. Lu, "Pythagorean fuzzy power aggregation operators in multiple attribute decision making," International Journal of Intelligent Systems, vol. 33, no. 1, pp. 169-186, 2018.

[21] Z. Yang and J. Chang, "Interval-valued Pythagorean normal fuzzy information aggregation operators for multi-attribute decision making," IEEE Access, vol. 8, pp. 51295-51314, 2020.

[22] S. Zeng, Z. Mu, and T. Baležentis, "A novel aggregation method for Pythagorean fuzzy multiple attribute group decision making," International Journal of Intelligent Systems, vol. 33, no. 3, pp. 573-585, 2018.

[23] S. Zeng, X. Peng, T. Baležentis, and D. Streimikiene, "Prioritization of low-carbon suppliers based on Pythagorean fuzzy group decision making with self-confidence level," Economic Research-Ekonomska Istraživanja, vol. 32, no. 1, pp. 1073-1087, 2019.

[24] S. Zeng, "Pythagorean fuzzy multiattribute group decision making with probabilistic information and OWA approach," International Journal of Intelligent Systems, vol. 32, no. 11, pp. 1136-1150, 2017.

[25] K. Ullah, T. Mahmood, Z. Ali, and N. Jan, "On some distance measures of complex Pythagorean fuzzy sets and their applications in pattern recognition," Complex and Intelligent Systems, vol. 6, no. 1, pp. 15-27, 2020.

[26] X. Peng, J. Dai, and H. Garg, "Exponential operation and aggregation operator for q-rung orthopair fuzzy set and their decision-making method with a new score function," International Journal of Intelligent Systems, vol. 33, no. 11, pp. 2255-2282, 2018.

[27] Z. Yang, T. Ouyang, X. Fu, and X. Peng, “A decision-making algorithm for online shopping using deep-learning-based opinion pairs mining and q-rung orthopair fuzzy interaction Heronian mean operators," International Journal of Intelligent Systems, vol. 35, no. 5, pp. 783-825, 2020.

[28] Z. L. Yang, H. Garg, J. Li, G. Srivastavad, and Z. Cao, "Investigation of multiple heterogeneous relationships using a q-rung orthopair fuzzy multi-criteria decision algorithm," Neural Computing and Applications, pp. 1-22. In press, 2020.

[29] A. Hussain, A. M. Irfan, and T. Mahmood, "Covering based q-rung orthopair fuzzy rough set model hybrid with TOPSIS for multi-attribute decision making," Journal of Intelligent and Fuzzy Systems, vol. 37, no. 1, pp. 981-993, 2019.

[30] A. Hussain, M. I. Ali, T. Mahmood, and M. Munir, "q-Rung orthopair fuzzy soft average aggregation operators and their application in multicriteria decision-making," International Journal of Intelligent Systems, vol. 35, no. 4, pp. 571-599, 2020.
[31] Z. Yang, X. Li, Z. Cao, and J. Li, "q-Rung orthopair normal fuzzy aggregation operators and their application in multi-attribute decision-making," Mathematics, vol. 7, no. 12, p. 1142, 2019.

[32] H. Garg, J. Gwak, T. Mahmood, and Z. Ali, "Power aggregation operators and VIKOR methods for complex q-rung orthopair fuzzy sets and their applications," Mathematics, vol. 8 , no. 4 , p. $538,2020$.

[33] P. Liu, Z. Ali, and T. Mahmood, "A method to multi-attribute group decision-making problem with complex q-rung orthopair linguistic information based on heronian mean operators," International Journal of Computational Intelligence Systems, vol. 12, no. 2, pp. 1465-1496, 2019.

[34] G. Wei, "Picture fuzzy Hamacher aggregation operators and their application to multiple attribute decision making," Fundamenta Informaticae, vol. 157, no. 3, pp. 271-320, 2018.

[35] S. Khan, S. Abdullah, L. Abdullah, and S. Ashraf, "Logarithmic aggregation operators of picture fuzzy numbers for multiattribute decision making problems," Mathematics, vol. 7, no. 7, p. $608,2019$.

[36] P. Liu, M. Munir, T. Mahmood, and K. Ullah, "Some similarity measures for interval-valued picture fuzzy sets and their applications in decision making," Information, vol. 10, no. 12, p. 369 , 2019.

[37] S. Zeng, S. Asharf, M. Arif, and S. Abdullah, "Application of exponential jensen picture fuzzy divergence measure in multicriteria group decision making," Mathematics, vol. 7, no. 2, p. 191, 2019.

[38] S. Zeng, D. Luo, C. Zhang, and X. Li, “A correlation-based TOPSIS method for multiple attribute decision making with single-valued neutrosophic information," International Journal of Information Technology \& Decision Making, vol. 19, no. 1, pp. 343-358, 2020.

[39] S. G. Quek, G. Selvachandran, M. Munir et al., "Multi-attribute multi-perception decision-making based on generalized T-spherical fuzzy weighted aggregation operators on neutrosophic sets," Mathematics, vol. 7, no. 9, p. 780, 2019.

[40] H. Garg, M. Munir, K. Ullah, T. Mahmood, and N. Jan, "Algorithm for T-spherical fuzzy multi-attribute decision making based on improved interactive aggregation operators," Symmetry, vol. 10, no. 12, p. 670, 2018.

[41] S. Zeng, H. Garg, M. Munir, T. Mahmood, and A. Hussain, "A multi-attribute decision making process with immediate probabilistic interactive averaging aggregation operators of T-spherical fuzzy sets and its application in the selection of solar cells," Energies, vol. 12, no. 23, p. 4436, 2019.

[42] M. Munir, H. Kalsoom, K. Ullah, T. Mahmood, and Y.-M. Chu, “T-spherical fuzzy Einstein hybrid aggregation operators and their applications in multi-attribute decision making problems," Symmetry, vol. 12, no. 3, p. 365, 2020.

[43] S. Zeng, A. Hussain, T. Mahmood, M. Irfan Ali, S. Ashraf, and M. Munir, "Covering-based spherical fuzzy rough set model hybrid with TOPSIS for multi-attribute decision-making," Symmetry, vol. 11, no. 4, p. 547, 2019.

[44] P. Liu, Q. Khan, T. Mahmood, and N. Hassan, “T-spherical fuzzy power Muirhead mean operator based on novel operational laws and their application in multi-attribute group decision making," IEEE Access, vol. 7, pp. 22613-22632, 2019.

[45] M. Q. Wu, T. Y. Chen, and J. P. Fan, "Divergence measure of T-spherical fuzzy sets and its applications in pattern recognition," IEEE Access, vol. 8, pp. 10208-10221, 2020.

[46] K. Ullah, H. Garg, T. Mahmood, N. Jan, and Z. Ali, "Correlation coefficients for T-spherical fuzzy sets and their applications in clustering and multi-attribute decision making," Soft Computing, vol. 24, no. 3, pp. 1647-1659, 2020. 